Supporting Information for

\title{
Insights into Catalytic Gas-Phase Hydrolysis of Organophosphate Chemical Warfare Agents by MOF-Supported Bimetallic Metal-Oxo Clusters
}

Haoyuan Chen and Randall Q. Snurr*

Department of Chemical \& Biological Engineering, Northwestern University, 2145 Sheridan Road, Evanston, IL 60208, USA

*Email: snurr@northwestern.edu

Full Atomistic Structures of Clusters
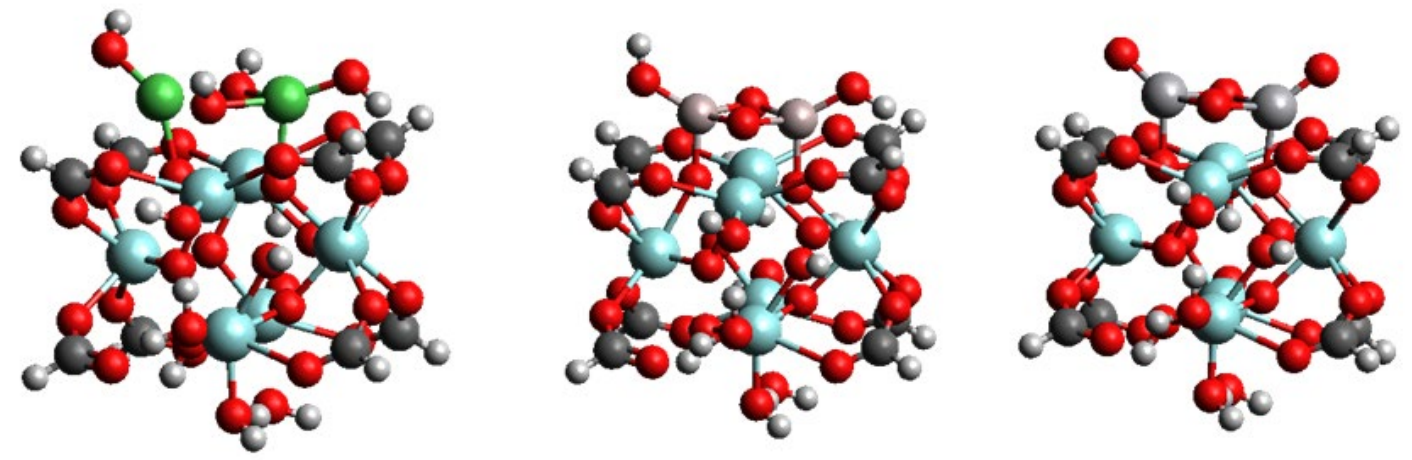

Figure S-1. Optimized structures of the NU-1000-supported bimetallic metal-oxo clusters with metals of +2 (Ni(II), left), +3 ( $\mathrm{Al}(\mathrm{III})$, middle) and +4 (V(IV), right) oxidation states, corresponding to Figure 2 in the main text. Color code: $\mathrm{H}$ - white, $\mathrm{C}$ - grey, $\mathrm{N}$-blue, $\mathrm{O}$-red, $\mathrm{Al}$ - pink, $\mathrm{Ni}$-green, $\mathrm{V}$ - silver, $\mathrm{Zr}$ cyan. 


\section{Full Atomistic Structures of DMPA Binding on Clusters}
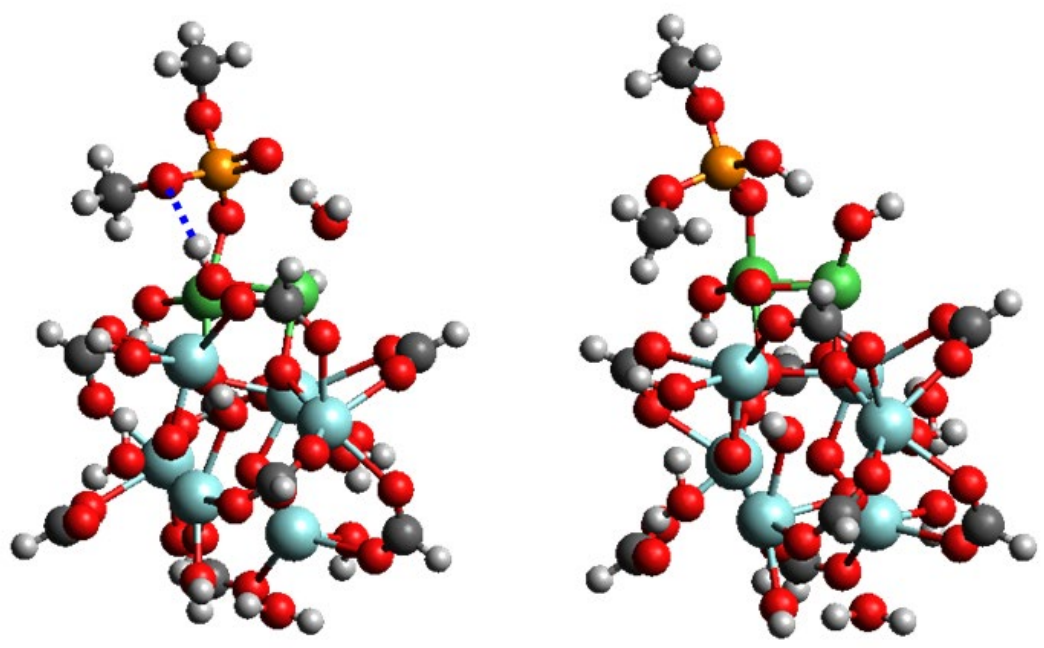

Figure S-2. Optimized structures of DMPA binding on NU-1000-supported bimetallic metal-oxo clusters with metals of +2 ( $\mathrm{Ni}(\mathrm{II})$, left) and +3 (Ni(III), right) oxidation states, corresponding to Figure 5 in the main text. The additional hydrogen bond in $\mathrm{Ni}(\mathrm{II})$ cluster is showed in blue. Color code: $\mathrm{H}-$ white, $\mathrm{C}-$ grey, $\mathrm{N}$-blue, $\mathrm{O}$-red, $\mathrm{P}$ - gold, $\mathrm{Ni}$ - green, $\mathrm{Zr}$ - cyan.

\section{Reaction Free Energy Profiles}

Table S-1. Original data used for plotting the reaction energy $(E)$ and free energy $(G)$ profiles in Figure 4 and calculating the TOFs in Table 2 in the main text. All values are in $\mathrm{kJ} / \mathrm{mol}$.

\begin{tabular}{|l|l|l|l|l|l|l|}
\hline & $\mathrm{NU}-1000$ & \multicolumn{2}{l}{$\mathrm{Al}_{2} @ \mathrm{NU}-1000$} & \multicolumn{2}{l|}{$\mathrm{Al}_{2} @ \mathrm{NU}-1000,+1 \mathrm{~W}$} \\
\cline { 2 - 7 } & $\mathrm{G}$ & $\mathrm{E}$ & $\mathrm{G}$ & $\mathrm{E}$ & $\mathrm{G}$ & $\mathrm{E}$ \\
\hline Initial & 0.0 & 0.0 & 0.0 & 0.0 & 0.0 & 0.0 \\
\hline DMNP Binding & -134.1 & -213.1 & -49.1 & -126.6 & -53.1 & -129.2 \\
\hline Pre-Reaction & -125.2 & -248.6 & -65.5 & -200.4 & -76.1 & -202.9 \\
\hline TS 1 & -41.1 & -171.0 & 98.4 & -34.1 & 70.3 & -58.7 \\
\hline INT 1 & -57.7 & -194.2 & 86.3 & -56.6 & 68.5 & -63.4 \\
\hline TS Berry & -37.2 & -174.7 & $\mathrm{~N} / \mathrm{A}$ & $\mathrm{N} / \mathrm{A}$ & $\mathrm{N} / \mathrm{A}$ & $\mathrm{N} / \mathrm{A}$ \\
\hline INT Berry & -74.8 & -211.8 & $\mathrm{~N} / \mathrm{A}$ & $\mathrm{N} / \mathrm{A}$ & $\mathrm{N} / \mathrm{A}$ & $\mathrm{N} / \mathrm{A}$ \\
\hline TS 2 & -74.8 & -211.9 & 97.2 & -43.9 & 73.0 & -64.7 \\
\hline DMPA Binding & -187.5 & -261.6 & -77.6 & -145.6 & -83.4 & -153.5 \\
\hline Final & -22.5 & -23.8 & -22.5 & -23.8 & -22.5 & -23.8 \\
\hline
\end{tabular}




\section{Potential Energy Scan of Berry Pseudorotation in Al2@NU-1000}

Table S-2. Potential energy scan of the Berry pseudorotation angle in $\mathrm{Al}_{2} @ \mathrm{NU}-1000$.

\begin{tabular}{|l|l|l|l|l|}
\hline Angle (degree) & 99 & 96 & 93 & 90 \\
\hline Relative Energy $(\mathrm{kJ} / \mathrm{mol})$ & 0.0 & -4.1 & -5.8 & -4.9 \\
\hline
\end{tabular}

A relaxed potential energy scan (all degrees of freedom were allowed to relax except for the scan coordinate) was performed on the $\mathrm{O}_{\mathrm{LG}}-\mathrm{P}-\mathrm{O}_{\mathrm{P}}$ angle, in which $\mathrm{O}_{\mathrm{LG}}$ is the phenol oxygen in the leaving group, $\mathrm{P}$ is the phosphorous and $\mathrm{O}_{\mathrm{P}}$ is the non-bridging oxygen on phosphorous. In TS 1 and INT 1, the angles are 99 and 93 degrees, respectively. In a complete Berry pseudorotation, this angle should go from 120 degree to 90 degree. Also, the potential energy goes monotonically downhill from 99 degrees to 93 degrees. These results suggest in this reaction, the Berry pseudorotation process occurs in a concerted fashion along with the nucleophilic attack. No separate transition state exists as in NU-1000 (TS Berry).

\section{Truncation of the Molecular Model}

Table S-3. Comparison of the water, DMNP and DMPA binding free energies on $\mathrm{Al}_{2} @ \mathrm{NU}-1000$ with benzoate and formate linkers. All values are in $\mathrm{kJ} / \mathrm{mol}$.

\begin{tabular}{|l|l|l|l|}
\hline & Water & DMNP & DMPA \\
\hline Benzoate Linker & -11.0 & -45.8 & -65.7 \\
\hline Formate Linker & -12.4 & -49.1 & -53.8 \\
\hline
\end{tabular}

In our previous work, ${ }^{1}$ the 8 linkers on a NU-1000 node were truncated to 4 benzoate (the 4 that are around the active site) and 4 formate (the other 4 that are farther away) groups. Here, to further reduce the computational cost, we truncated all 8 linkers on the node to formate groups. The binding free energies of water, DMNP and DMPA using formate and benzoate cappings were compared and no significant differences were observed. Therefore, the formate capping was used for mapping out the complete reaction pathway. 


\section{Optimized Cartesian Coordinates of the Key Species (distance unit is Angstrom)}

\begin{tabular}{|c|c|c|c|}
\hline \multicolumn{4}{|c|}{ NU-1000 Initial Node } \\
\hline $\mathrm{C}$ & 5.69635300 & -2.73268500 & -1.97500200 \\
\hline $\mathrm{C}$ & -5.67275700 & -2.69913900 & -1.98945800 \\
\hline $\mathrm{C}$ & 4.35122900 & -3.00031500 & -2.27015600 \\
\hline $\mathrm{C}$ & -4.33468800 & -2.94694200 & -2.33093800 \\
\hline $\mathrm{C}$ & 4.04531400 & -3.97495100 & -3.23161000 \\
\hline $\mathrm{C}$ & -4.05084100 & -3.93326800 & -3.28730700 \\
\hline $\mathrm{C}$ & 5.06214300 & -4.68750000 & -3.85997100 \\
\hline $\mathrm{C}$ & -5.07729000 & -4.67687000 & -3.86317500 \\
\hline $\mathrm{C}$ & 6.41474500 & -4.45091300 & -3.54603300 \\
\hline $\mathrm{C}$ & -6.42201800 & -4.45432500 & -3.50679400 \\
\hline $\mathrm{C}$ & 6.71064700 & -3.44497300 & -2.60711200 \\
\hline $\mathrm{C}$ & -6.69792500 & -3.43779700 & -2.57215000 \\
\hline $\mathrm{C}$ & 5.65210700 & 2.91189700 & -1.62887800 \\
\hline $\mathrm{C}$ & -5.69468600 & 2.81121000 & -1.68112600 \\
\hline $\mathrm{C}$ & 4.31248400 & 3.20500600 & -1.92469800 \\
\hline $\mathrm{C}$ & -4.35830100 & 3.08471900 & -2.01159500 \\
\hline $\mathrm{C}$ & 4.02071900 & 4.28938600 & -2.76447200 \\
\hline $\mathrm{C}$ & -4.07204100 & 4.20261400 & -2.81002300 \\
\hline $\mathrm{C}$ & 5.04481700 & 5.08324500 & -3.27180700 \\
\hline $\mathrm{C}$ & -5.09649300 & 5.03777500 & -3.24983000 \\
\hline $\mathrm{C}$ & 6.39154700 & 4.81382800 & -2.95945600 \\
\hline $\mathrm{C}$ & -6.44002700 & & -2.91583600 \\
\hline $\mathrm{C}$ & 6.67478400 & 3.70202200 & -2.14386500 \\
\hline $\mathrm{C}$ & -6.71749500 & 3.64134000 & -2.13059500 \\
\hline $\mathrm{C}$ & -3.24821100 & -2.18275700 & -1.65008300 \\
\hline $\mathrm{C}$ & -3.22969400 & -2.38658000 & 2.90001900 \\
\hline $\mathrm{C}$ & -3.23228900 & 2.03429600 & 3.10403700 \\
\hline $\mathrm{C}$ & 3.27688900 & 2.14200100 & 2.93925000 \\
\hline $\mathrm{C}$ & 3.22685000 & 2.36634900 & -1.33869500 \\
\hline $\mathrm{C}$ & 3.25925400 & -2.27794200 & -1.56154800 \\
\hline $\mathrm{C}$ & -3.26527400 & 2.23609200 & -1.45725500 \\
\hline $\mathrm{C}$ & 3.25722500 & -2.50655600 & 2.71639600 \\
\hline $\mathrm{H}$ & 5.94154000 & -1.96564800 & -1.23798700 \\
\hline $\mathrm{H}$ & -5.90552100 & -1.92630600 & -1.25515300 \\
\hline $\mathrm{H}$ & 3.00124900 & -4.17887100 & -3.47567800 \\
\hline $\mathrm{H}$ & -3.01276100 & -4.12404500 & -3.56755000 \\
\hline $\mathrm{H}$ & 4.80563900 & -5.45081700 & -4.59906000 \\
\hline $\mathrm{H}$ & -4.83354000 & -5.44923000 & -4.59710800 \\
\hline $\mathrm{H}$ & 7.75317400 & -3.22900300 & -2.35971400 \\
\hline $\mathrm{H}$ & -7.73398200 & -3.23444500 & -2.28901000 \\
\hline $\mathrm{H}$ & 5.88535700 & 2.06263800 & -0.98453200 \\
\hline
\end{tabular}




\begin{tabular}{|c|c|c|c|}
\hline $\mathrm{H}$ & -5.92614600 & 1.94451700 & -1.05922700 \\
\hline $\mathrm{H}$ & 2.98038600 & 4.51428400 & -3.00859500 \\
\hline $\mathrm{H}$ & -3.03540900 & 4.42245900 & -3.07212300 \\
\hline $\mathrm{H}$ & 4.79958500 & 5.92995200 & -3.91782700 \\
\hline $\mathrm{H}$ & -4.85230700 & 5.90921400 & -3.86256800 \\
\hline $\mathrm{H}$ & 7.71307000 & 3.46432500 & -1.89878600 \\
\hline $\mathrm{H}$ & -7.75183100 & 3.41281300 & -1.86098200 \\
\hline $\mathrm{H}$ & -7.22636000 & -5.04165500 & -3.95292400 \\
\hline $\mathrm{H}$ & 7.21092900 & -5.01777500 & -4.03160300 \\
\hline $\mathrm{H}$ & -7.24177100 & 5.43320700 & -3.25857500 \\
\hline $\mathrm{H}$ & 7.19410500 & 5.44034300 & -3.35245600 \\
\hline $\mathrm{H}$ & -1.92675600 & -2.95200100 & 0.60997900 \\
\hline $\mathrm{H}$ & -0.92438600 & 1.44324400 & 5.00966900 \\
\hline $\mathrm{H}$ & -0.04924000 & 0.14476100 & 4.79073700 \\
\hline $\mathrm{H}$ & 0.04096600 & 4.02474100 & 0.52003000 \\
\hline $\mathrm{H}$ & 0.72059500 & 4.26565400 & 2.61569600 \\
\hline $\mathrm{H}$ & 0.88793900 & 4.27909100 & -0.78474600 \\
\hline $\mathrm{H}$ & 1.95909900 & -0.17074600 & 3.57622300 \\
\hline $\mathrm{H}$ & -1.93695000 & 2.80014300 & 0.82450300 \\
\hline $\mathrm{H}$ & -0.65270700 & -1.97852300 & 5.09134700 \\
\hline $\mathrm{H}$ & 0.93761000 & -4.37805800 & 2.21209900 \\
\hline $\mathrm{H}$ & 0.71000900 & -4.39722000 & -1.16064800 \\
\hline $\mathrm{H}$ & 0.05540300 & -4.14973800 & 0.92142200 \\
\hline $\mathrm{O}$ & -1.07199000 & -0.09131000 & 2.18651200 \\
\hline $\mathrm{O}$ & -3.62507300 & 1.32085700 & 2.14778800 \\
\hline $\mathrm{O}$ & -3.60722600 & 1.32618900 & -0.64627900 \\
\hline $\mathrm{O}$ & -3.61297400 & -1.51370900 & 2.08731300 \\
\hline $\mathrm{O}$ & -2.05362600 & -2.65285900 & 3.25149700 \\
\hline $\mathrm{O}$ & 0.06207700 & -4.08475400 & 1.92627500 \\
\hline $\mathrm{O}$ & 2.08144100 & -2.82168700 & 3.01928600 \\
\hline $\mathrm{O}$ & 2.05778600 & -2.56905700 & -1.86602400 \\
\hline $\mathrm{O}$ & 3.63474100 & -1.52360900 & 2.03457000 \\
\hline $\mathrm{O}$ & 3.59558900 & -1.42612900 & -0.69706400 \\
\hline $\mathrm{O}$ & 3.64072100 & 1.28166600 & 2.09801600 \\
\hline $\mathrm{O}$ & 3.58317400 & 1.34941800 & -0.66622100 \\
\hline $\mathrm{O}$ & 2.03774000 & 2.70903400 & -1.54879700 \\
\hline $\mathrm{O}$ & 2.11046300 & 2.46645900 & 3.25325700 \\
\hline $\mathrm{O}$ & 1.07986100 & 1.38259500 & 0.71815500 \\
\hline $\mathrm{O}$ & 1.40056600 & -0.11286800 & 2.79483300 \\
\hline $\mathrm{O}$ & 0.02500700 & -1.51247600 & 4.59271900 \\
\hline $\mathrm{O}$ & -0.05302000 & 1.14743100 & 4.71424100 \\
\hline $\mathrm{O}$ & 1.06985200 & -1.55277800 & 0.69917000 \\
\hline $\mathrm{O}$ & -1.38837400 & -2.15842100 & 0.69200500 \\
\hline $\mathrm{O}$ & -2.04529500 & -2.36350700 & -2.00656800 \\
\hline
\end{tabular}




$\begin{array}{lrrr}\mathrm{O} & -0.00823700 & -3.93169800 & -0.72154000 \\ \mathrm{O} & -3.60676200 & -1.41277300 & -0.70830100 \\ \mathrm{O} & 0.01519600 & 3.79173900 & 2.16527400 \\ \mathrm{O} & 0.02036800 & 3.97763600 & -0.48395600 \\ \mathrm{O} & -1.38431500 & 2.01612700 & 0.74677800 \\ \mathrm{O} & -2.04996000 & 2.29922600 & 3.42525000 \\ \mathrm{O} & -2.08586500 & 2.49013500 & -1.81380500 \\ \mathrm{O} & 1.31924100 & -0.04996400 & -1.45564900 \\ \mathrm{O} & -1.07611400 & -0.05197500 & -0.76031200 \\ \mathrm{Zr} & 2.52830700 & -0.05056200 & 0.69563500 \\ \mathrm{Zr} & -0.03556000 & -1.85055200 & 2.53847800 \\ \mathrm{Zr} & 0.04541700 & 1.73084800 & 2.47644600 \\ \mathrm{Zr} & -0.05350700 & 1.74938600 & -1.13882300 \\ \mathrm{Zr} & -2.53183900 & -0.05988900 & 0.70092500 \\ \mathrm{Zr} & 0.02897900 & -1.85696400 & -1.00721500 \\ \mathrm{H} & 4.11539800 & 2.67904000 & 3.44567600 \\ \mathrm{H} & 4.06668700 & -3.18352700 & 3.08659200 \\ \mathrm{H} & -4.03009300 & 2.43761800 & 3.77314700 \\ \mathrm{H} & -4.03201900 & -2.99069700 & 3.38887000 \\ \mathrm{H} & 1.87497100 & 0.01939300 & -2.23807400 \\ \mathrm{O} & 0.07731100 & 1.70775200 & -3.14605300 \\ \mathrm{H} & -0.71466200 & 1.93517300 & -3.63928700\end{array}$

$\begin{array}{lrrr}\text { NU-1000 DMNP Binding } & & \\ \mathrm{C} & 5.71834500 & 0.43210500 & -2.15072500 \\ \mathrm{C} & -4.97460100 & 4.15699400 & -1.12892000 \\ \mathrm{C} & 4.57174900 & 1.24032600 & -2.13366800 \\ \mathrm{C} & -3.58343400 & 4.11413300 & -1.30549000 \\ \mathrm{C} & 4.67920600 & 2.58395400 & -2.52214600 \\ \mathrm{C} & -2.92190200 & 5.26563900 & -1.75775100 \\ \mathrm{C} & 5.90012100 & 3.09812700 & -2.94817000 \\ \mathrm{C} & -3.63792100 & 6.42111100 & -2.05836700 \\ \mathrm{C} & 7.05004200 & 2.28642900 & -3.00196100 \\ \mathrm{C} & -5.03825800 & 6.46560100 & -1.91036600 \\ \mathrm{C} & 6.93808600 & 0.95006700 & -2.57458700 \\ \mathrm{C} & -5.68756000 & 5.31537500 & -1.42210400 \\ \mathrm{C} & 5.10873200 & -2.45657300 & 2.67287500 \\ \mathrm{C} & -5.53994700 & 1.35444100 & 3.59181000 \\ \mathrm{C} & 3.92256900 & -1.90959800 & 3.18438100 \\ \mathrm{C} & -4.19584700 & 1.06134300 & 3.87018900 \\ \mathrm{C} & 3.83753000 & -1.62596900 & 4.55505900 \\ \mathrm{C} & -3.75338400 & 1.10587900 & 5.20109800 \\ \mathrm{C} & 4.90427800 & -1.91250000 & 5.40141800 \\ \mathrm{C} & -4.63931700 & 1.41640000 & 6.23023800\end{array}$




\begin{tabular}{|c|c|c|c|}
\hline $\mathrm{C}$ & 6.08988700 & -2.48625000 & 900 \\
\hline C & -5.99228500 & 1.70312500 & 5.96206400 \\
\hline C & 6.17696000 & -2.73462200 & 3.51960500 \\
\hline C & -6.42075400 & 1.67467100 & 4.62084500 \\
\hline C & -2.84718000 & 2.84312000 & -1.04081100 \\
\hline C & -4.31313100 & -0.81287900 & -3.32730100 \\
\hline C & -4.73815600 & -3.03074400 & 0.47876700 \\
\hline C & 1.43090200 & -5.07995400 & 0.08562500 \\
\hline C & 2.77565700 & -1.64686700 & 2.26803600 \\
\hline $\mathrm{C}$ & 3.25314500 & 0.68005000 & -1.72981100 \\
\hline $\mathrm{C}$ & -3.28230100 & 0.63974800 & 2.77021400 \\
\hline C & 1.85961900 & -2.73368600 & -3.91077400 \\
\hline $\mathrm{H}$ & 5.64442700 & -0.61065000 & -1.83631600 \\
\hline $\mathrm{H}$ & -5.49770100 & 3.27053200 & -0.76669500 \\
\hline $\mathrm{H}$ & 3.79387700 & 3.22181200 & -2.49860600 \\
\hline $\mathrm{H}$ & -1.83768000 & 5.24431300 & -1.88639600 \\
\hline $\mathrm{H}$ & 5.96377400 & 4.14456900 & -3.25737300 \\
\hline $\mathrm{H}$ & -3.10464700 & 7.30344400 & -2.42129900 \\
\hline $\mathrm{H}$ & 7.81990200 & 0.30438400 & -2.58889800 \\
\hline $\mathrm{H}$ & -6.77184800 & 5.32781200 & -1.28390300 \\
\hline $\mathrm{H}$ & 5.18411400 & -2.66937400 & 1.60523100 \\
\hline $\mathrm{H}$ & -5.89294300 & 1.32053200 & 2.55946900 \\
\hline $\mathrm{H}$ & 2.92126300 & -1.18815500 & 4.95661200 \\
\hline $\mathrm{H}$ & -2.70952200 & 0.87963100 & 5.42647800 \\
\hline $\mathrm{H}$ & 4.81839300 & -1.69317400 & 6.46861600 \\
\hline $\mathrm{H}$ & -4.27776000 & 1.43635600 & 7.26143000 \\
\hline $\mathrm{H}$ & 7.09203300 & -3.16656800 & 3.10648700 \\
\hline $\mathrm{H}$ & -7.46423500 & 1.89800900 & 4.38413100 \\
\hline $\mathrm{H}$ & -5.60065700 & 7.36897900 & -2.15237600 \\
\hline $\mathrm{H}$ & 8.00462100 & 2.68787100 & -3.34640500 \\
\hline $\mathrm{H}$ & -6.68631400 & 1.94372600 & 6.76911800 \\
\hline $\mathrm{H}$ & 6.92399200 & -2.71664100 & 5.56747400 \\
\hline $\mathrm{H}$ & -2.29909400 & 0.90205100 & -2.85738500 \\
\hline $\mathrm{H}$ & -3.14720800 & -5.07329200 & -1.15575300 \\
\hline $\mathrm{H}$ & -2.13929500 & -4.55981200 & -2.26344600 \\
\hline $\mathrm{H}$ & -0.96818500 & -2.92335000 & 3.14567000 \\
\hline $\mathrm{H}$ & -1.04565100 & -4.97317100 & 2.32773100 \\
\hline $\mathrm{H}$ & 0.24862000 & -2.24614900 & 3.88706900 \\
\hline $\mathrm{H}$ & 0.16536400 & -4.09075100 & -2.14154200 \\
\hline $\mathrm{H}$ & -2.82525000 & -1.94442700 & 2.10811200 \\
\hline $\mathrm{H}$ & -2.62912000 & -3.60231300 & -4.19787300 \\
\hline $\mathrm{H}$ & 0.01917000 & -0.62933600 & -5.09767800 \\
\hline $\mathrm{H}$ & 0.96323800 & 2.10268400 & -3.50213200 \\
\hline $\mathrm{H}$ & -0.41304600 & 0.60189600 & -4.20303700 \\
\hline
\end{tabular}




$\begin{array}{lrrr}\mathrm{O} & -2.22794900 & -1.99553600 & -1.14903500 \\ \mathrm{O} & -4.73356500 & -1.78246300 & 0.33226300 \\ \mathrm{O} & -3.79925700 & 0.50923100 & 1.62477700 \\ \mathrm{O} & -4.47903300 & -0.42324000 & -2.14570800 \\ \mathrm{O} & -3.29227000 & -1.34600400 & -3.82410700 \\ \mathrm{O} & -0.73751300 & -0.25313600 & -4.62873600 \\ \mathrm{O} & 0.68270400 & -2.43474200 & -4.22607800 \\ \mathrm{O} & 2.25108200 & 1.44521800 & -1.78138100 \\ \mathrm{O} & 2.36295900 & -2.75571600 & -2.76200600 \\ \mathrm{O} & 3.23167200 & -0.53277900 & -1.37877300 \\ \mathrm{O} & 2.11725400 & -4.10860600 & -0.31388600 \\ \mathrm{O} & 2.97227000 & -1.86587700 & 1.03692800 \\ \mathrm{O} & 1.70145200 & -1.23584000 & 2.77533300 \\ \mathrm{O} & 0.20148600 & -5.11250200 & 0.32424100 \\ \mathrm{O} & 0.15341500 & -2.20815000 & 0.65118400 \\ \mathrm{O} & -0.10113700 & -3.29415400 & -1.67237800 \\ \mathrm{O} & -1.86334200 & -3.64733400 & -3.61758900 \\ \mathrm{O} & -2.20529200 & -4.97651800 & -1.34768200 \\ \mathrm{O} & 0.40021900 & -0.79158400 & -1.92341700 \\ \mathrm{O} & -1.86580300 & 0.31124400 & -2.23404200 \\ \mathrm{O} & -1.59561400 & 2.84510600 & -1.18216400 \\ \mathrm{O} & 0.05971900 & 1.87786300 & -3.25549500 \\ \mathrm{O} & -3.54902400 & 1.83774400 & -0.72705700 \\ \mathrm{H} & -0.18944500 & 1.54548700 & 3.47504000 \\ \mathrm{O} & 0.76443500 & 2.73909700 & 0.06667600 \\ \mathrm{H} & -1.52675900 & -4.15304400 & 2.18199200 \\ \mathrm{O} & -0.64734000 & -2.06878000 & 3.57046300 \\ \mathrm{O} & -2.22175300 & -1.69615500 & 1.40100000 \\ \mathrm{O} & -3.75568200 & -3.80744800 & 0.45519200 \\ \mathrm{O} & -2.07645200 & 0.42429400 & 3.06109300 \\ \mathrm{O} & 1.22615700 & 0.10195500 & 0.36055300 \\ \mathrm{Zr} & -5.18007200 & -0.67211100 & -4.01716300 \\ \mathrm{Or} & -1.27626500 & 0.42560500 & 0.27157000 \\ \mathrm{Zr} & 1.64494900 & -1.96051900 & -0.75191400 \\ \mathrm{Zr} & -1.20409700 & -1.77890900 & -2.94789800 \\ \mathrm{Zr} & -1.42179500 & -3.46224400 & 0.21429600 \\ \mathrm{H} & -0.32310500 & -0.48813500 & 1.89979000 \\ \mathrm{H} & -3.11328600 & -0.32142000 & -0.31993400 \\ \mathrm{H} & 2.00956400 & -6.02075000 & 0.25253300 \\ \mathrm{H} & -5.74145300 & -3.50694900 & 0.59404200 \\ \mathrm{O} & & & \end{array}$




$\begin{array}{lrrr}\mathrm{P} & 0.74001700 & 4.07334600 & 0.69773900 \\ \mathrm{O} & 1.11700500 & 5.22446500 & -0.29722900 \\ \mathrm{O} & -0.59090900 & 4.53815700 & 1.34492100 \\ \mathrm{O} & 1.81407900 & 4.17084200 & 1.85812600 \\ \mathrm{C} & 1.08560900 & 4.95691900 & -1.71315800 \\ \mathrm{H} & 1.11467800 & 5.92311200 & -2.20718300 \\ \mathrm{H} & 1.95827500 & 4.36538700 & -1.99067900 \\ \mathrm{H} & 0.18490200 & 4.41086300 & -1.99665900 \\ \mathrm{C} & -1.51700800 & 3.60810500 & 1.94506800 \\ \mathrm{H} & -2.51260000 & 4.00185800 & 1.75520500 \\ \mathrm{H} & -1.41468900 & 2.61498300 & 1.50472500 \\ \mathrm{H} & -1.33423500 & 3.56627300 & 3.01738200 \\ \mathrm{C} & 3.04924800 & 3.55917900 & 1.63083600 \\ \mathrm{C} & 4.04634400 & 4.27475500 & 0.98754000 \\ \mathrm{C} & 3.23205100 & 2.25355400 & 2.06431100 \\ \mathrm{C} & 5.26591000 & 3.66269000 & 0.75806400 \\ \mathrm{H} & 3.85885600 & 5.29203500 & 0.67188300 \\ \mathrm{C} & 4.44759100 & 1.63966600 & 1.81468400 \\ \mathrm{H} & 2.41250600 & 1.74764600 & 2.57144400 \\ \mathrm{C} & 5.44372200 & 2.35187600 & 1.16543600 \\ \mathrm{H} & 6.07440400 & 4.17445500 & 0.25661300 \\ \mathrm{H} & 4.63988700 & 0.61874000 & 2.11643800 \\ \mathrm{~N} & 6.72954600 & 1.69122300 & 0.89143400 \\ \mathrm{O} & 7.62014300 & 2.37233200 & 0.40858000 \\ \mathrm{O} & 6.82077700 & 0.50508600 & 1.16046600\end{array}$

$\begin{array}{lrrr}\text { NU-1000 Pre-Reaction Complex } & \\ \mathrm{C} & 5.68547300 & 0.06624500 & -2.26049100 \\ \mathrm{C} & -4.92374300 & 4.08742500 & -1.53067000 \\ \mathrm{C} & 4.55502800 & 0.89537900 & -2.31363100 \\ \mathrm{C} & -3.53523600 & 3.99969300 & -1.71153800 \\ \mathrm{C} & 4.68531200 & 2.19304300 & -2.83024700 \\ \mathrm{C} & -2.85529100 & 5.08883800 & -2.27684300 \\ \mathrm{C} & 5.91232400 & 2.63914200 & -3.31185500 \\ \mathrm{C} & -3.55120900 & 6.22433800 & -2.68243900 \\ \mathrm{C} & 7.04570900 & 1.80295300 & -3.29496300 \\ \mathrm{C} & -4.94909500 & 6.31113100 & -2.53010100 \\ \mathrm{C} & 6.91140100 & 0.51634600 & -2.74030100 \\ \mathrm{C} & -5.61648200 & 5.22647200 & -1.92916800 \\ \mathrm{C} & 5.06099400 & -2.33294600 & 2.82253100 \\ \mathrm{C} & -5.50316600 & 1.76317000 & 3.44138400 \\ \mathrm{C} & 3.89013300 & -1.71551400 & 3.28692800 \\ \mathrm{C} & -4.16269800 & 1.47105200 & 3.73772600 \\ \mathrm{C} & 3.82236700 & -1.29990500 & 4.62450400\end{array}$




\begin{tabular}{|c|c|c|c|}
\hline $\mathrm{C}$ & -3.70808100 & 1.63423900 & 5.05521100 \\
\hline $\mathrm{C}$ & 4.89053200 & -1.52533900 & 5.48739200 \\
\hline $\mathrm{C}$ & -4.57895700 & 2.05999900 & 6.05557600 \\
\hline $\mathrm{C}$ & 6.06042600 & -2.16820100 & 5.03805500 \\
\hline C & -5.92832800 & 2.34693500 & 5.77006400 \\
\hline $\mathrm{C}$ & 6.13080800 & -2.54993300 & 3.68496400 \\
\hline $\mathrm{C}$ & -6.36873100 & 2.19848100 & 4.44067600 \\
\hline $\mathrm{C}$ & -2.82164500 & 2.74534800 & -1.33073100 \\
\hline $\mathrm{C}$ & -4.37805200 & -1.08294400 & -3.24518600 \\
\hline $\mathrm{C}$ & -4.81362400 & -2.91600700 & 0.75929900 \\
\hline $\mathrm{C}$ & 1.31075000 & -5.11777900 & 0.52409800 \\
\hline $\mathrm{C}$ & 2.74075600 & -1.51886700 & 2.35720100 \\
\hline $\mathrm{C}$ & 3.22926100 & 0.40324600 & -1.84900400 \\
\hline $\mathrm{C}$ & -3.26698200 & 0.92739000 & 2.67735100 \\
\hline $\mathrm{C}$ & 1.75088500 & -3.17528500 & -3.68220400 \\
\hline $\mathrm{H}$ & 5.59394800 & -0.93978800 & -1.84675900 \\
\hline $\mathrm{H}$ & -5.46089400 & 3.25060100 & -1.08139400 \\
\hline $\mathrm{H}$ & 3.81281200 & 2.84798000 & -2.86228700 \\
\hline $\mathrm{H}$ & -1.77283200 & 5.03334500 & -2.41004200 \\
\hline $\mathrm{H}$ & 5.99370300 & 3.64955400 & -3.72069000 \\
\hline $\mathrm{H}$ & -3.00397200 & 7.05677800 & -3.13208900 \\
\hline $\mathrm{H}$ & 7.78031700 & -0.14541900 & -2.69830000 \\
\hline $\mathrm{H}$ & -6.69909900 & 5.27403800 & -1.78560200 \\
\hline $\mathrm{H}$ & 5.12307900 & -2.64877200 & 1.77983000 \\
\hline $\mathrm{H}$ & -5.86557700 & 1.63742300 & 2.41945800 \\
\hline $\mathrm{H}$ & 2.91827300 & -0.80712100 & 4.98816500 \\
\hline $\mathrm{H}$ & -2.66693400 & 1.40962500 & 5.29436800 \\
\hline $\mathrm{H}$ & 4.81807100 & -1.20285000 & 6.52909400 \\
\hline $\mathrm{H}$ & -4.20827100 & 2.17157800 & 7.07764400 \\
\hline $\mathrm{H}$ & 7.03372400 & -3.03796200 & 3.30923700 \\
\hline $\mathrm{H}$ & -7.40965100 & 2.41909900 & 4.19051600 \\
\hline $\mathrm{H}$ & -5.49583900 & 7.19827700 & -2.85413900 \\
\hline $\mathrm{H}$ & 8.00494600 & 2.15008400 & -3.68275600 \\
\hline $\mathrm{H}$ & -6.61060700 & 2.67790800 & 6.55482800 \\
\hline $\mathrm{H}$ & 6.89554500 & -2.35047700 & 5.71660100 \\
\hline $\mathrm{H}$ & -2.32873700 & 0.63267600 & -2.94465600 \\
\hline $\mathrm{H}$ & -3.28014100 & -5.14955400 & -0.68723000 \\
\hline $\mathrm{H}$ & -2.27266000 & -4.75788300 & -1.8439600 \\
\hline $\mathrm{H}$ & -1.01377800 & -2.64715700 & 3.3733900 \\
\hline $\mathrm{H}$ & -1.14344000 & -4.75618700 & 2.7549000 \\
\hline $\mathrm{H}$ & 0.20878900 & -1.91735000 & 4.06039000 \\
\hline $\mathrm{H}$ & 0.04022600 & -4.32608300 & -1.7841470 \\
\hline $\mathrm{H}$ & -2.86092000 & -1.72052000 & 2.2665270 \\
\hline $\mathrm{H}$ & -2.75595400 & -3.98049200 & -3.8589920 \\
\hline
\end{tabular}




\begin{tabular}{|c|c|c|c|}
\hline $\mathrm{H}$ & -0.07036900 & -1.15384900 & -5.06380800 \\
\hline $\mathrm{H}$ & 0.94863300 & 1.68646600 & -3.73607200 \\
\hline $\mathrm{H}$ & -0.46367500 & 0.16112800 & -4.27617000 \\
\hline $\mathrm{O}$ & -2.29749000 & -2.09250300 & -0.97667700 \\
\hline $\mathrm{O}$ & -4.78510600 & -1.68860000 & 0.49138400 \\
\hline $\mathrm{O}$ & -3.79466500 & 0.69885900 & 1.55185300 \\
\hline $\mathrm{O}$ & -4.52605700 & -0.57880300 & -2.10535100 \\
\hline $\mathrm{O}$ & -3.37197200 & -1.68236600 & -3.6950940 \\
\hline $\mathrm{O}$ & -0.81027500 & -0.72348300 & -4.6159200 \\
\hline $\mathrm{O}$ & 0.57747800 & -2.88534700 & -4.01664400 \\
\hline $\mathrm{O}$ & 2.24044200 & 1.17869100 & -1.96750500 \\
\hline $\mathrm{O}$ & 2.26366500 & -3.09432200 & -2.53977500 \\
\hline $\mathrm{O}$ & 3.18835400 & -0.76745500 & -1.37745700 \\
\hline $\mathrm{O}$ & 2.01248200 & -4.20352600 & 0.02824700 \\
\hline $\mathrm{O}$ & 2.91913500 & -1.86600800 & 1.15315200 \\
\hline $\mathrm{O}$ & 1.68242000 & -1.02577500 & 2.82585400 \\
\hline $\mathrm{O}$ & 0.08264600 & -5.10340300 & 0.77033300 \\
\hline $\mathrm{O}$ & 0.09354700 & -2.18411700 & 0.81974100 \\
\hline $\mathrm{O}$ & -0.20512000 & -3.48343800 & -1.38975800 \\
\hline $\mathrm{O}$ & -1.98806100 & -3.98340400 & -3.27969100 \\
\hline $\mathrm{O}$ & -2.33843800 & -5.08307800 & -0.89177100 \\
\hline $\mathrm{O}$ & 0.34402500 & -1.02536300 & -1.87888500 \\
\hline $\mathrm{O}$ & -1.90002500 & 0.08998800 & -2.27601600 \\
\hline $\mathrm{O}$ & -1.56950500 & 2.70967700 & -1.47173200 \\
\hline $\mathrm{O}$ & 0.04335200 & 1.50836200 & -3.45974700 \\
\hline $\mathrm{O}$ & -3.54006600 & 1.78661500 & -0.92317400 \\
\hline $\mathrm{O}$ & -1.60866900 & -3.94296400 & 2.53702100 \\
\hline $\mathrm{O}$ & -0.67768000 & -1.75907700 & 3.70995200 \\
\hline $\mathrm{O}$ & -2.26246300 & -1.55532900 & 1.53126100 \\
\hline $\mathrm{O}$ & -3.84653600 & -3.71134000 & 0.80528400 \\
\hline $\mathrm{O}$ & -2.06417000 & 0.70616800 & 2.97847700 \\
\hline $\mathrm{O}$ & 1.20870500 & 0.06407500 & 0.30058700 \\
\hline $\mathrm{O}$ & -1.28442200 & 0.43511400 & 0.20507200 \\
\hline $\mathrm{Zr}$ & 1.57732600 & -2.10256500 & -0.61293500 \\
\hline $\mathrm{Zr}$ & -1.28778900 & -2.07362200 & -2.79625100 \\
\hline $\mathrm{Zr}$ & -1.51244500 & -3.44317600 & 0.51203800 \\
\hline $\mathrm{Zr}$ & -0.33493300 & -0.35286200 & 1.90137100 \\
\hline $\mathrm{Zr}$ & -3.14304500 & -0.32803000 & -0.30384900 \\
\hline $\mathrm{Zr}$ & -0.00117500 & 0.97466600 & -1.4178920 \\
\hline $\mathrm{H}$ & 1.87238300 & -6.04970200 & 0.77687400 \\
\hline $\mathrm{H}$ & 2.43247200 & -3.52478000 & -4.49686400 \\
\hline $\mathrm{H}$ & -5.82498300 & -3.35856400 & 0.9265040 \\
\hline $\mathrm{H}$ & -5.24795900 & -0.99158100 & -3.9396140 \\
\hline $\mathrm{H}$ & 1.98283600 & 0.57539200 & 0.5586050 \\
\hline
\end{tabular}




$\begin{array}{lrrr}\mathrm{O} & 0.43932800 & 1.36534800 & 2.75181500 \\ \mathrm{H} & -0.30205400 & 1.79682400 & 3.19320000 \\ \mathrm{O} & 0.80737900 & 2.66962700 & -0.23341900 \\ \mathrm{P} & 0.84323400 & 4.08063100 & 0.19730400 \\ \mathrm{O} & 1.20326900 & 5.07401200 & -0.95767000 \\ \mathrm{O} & -0.43075300 & 4.67114400 & 0.84867500 \\ \mathrm{O} & 1.98719500 & 4.29971100 & 1.28248200 \\ \mathrm{C} & 1.14566900 & 4.60757600 & -2.32141200 \\ \mathrm{H} & 1.26670000 & 5.48614000 & -2.94708800 \\ \mathrm{H} & 1.95635500 & 3.90044700 & -2.49970900 \\ \mathrm{H} & 0.19612700 & 4.11465000 & -2.53263100 \\ \mathrm{C} & -1.34277200 & 3.85417500 & 1.62416300 \\ \mathrm{H} & -2.32568900 & 4.30008500 & 1.49760400 \\ \mathrm{H} & -1.34494000 & 2.82788600 & 1.25175900 \\ \mathrm{H} & -1.03867800 & 3.88523000 & 2.67009700 \\ \mathrm{C} & 3.19139200 & 3.61222900 & 1.10876400 \\ \mathrm{C} & 4.22491400 & 4.23176300 & 0.42665200 \\ \mathrm{C} & 3.31542600 & 2.33943900 & 1.64846300 \\ \mathrm{C} & 5.42398700 & 3.55704100 & 0.27575100 \\ \mathrm{H} & 4.08352600 & 5.22620000 & 0.02593300 \\ \mathrm{C} & 4.50966600 & 1.66080900 & 1.47847600 \\ \mathrm{H} & 2.47647900 & 1.90425200 & 2.18541300 \\ \mathrm{C} & 5.54416600 & 2.28057400 & 0.79601600 \\ \mathrm{C} & -5.01047900 & 6.62599300 & -1.54411300 \\ \mathrm{H} & 6.26148400 & 3.99532100 & -0.24721500 \\ \mathrm{H} & 4.65668800 & 0.66277900 & 1.86863900 \\ \mathrm{~N} & 6.81297700 & 1.55533700 & 0.61540500 \\ \mathrm{O} & 7.73738300 & 2.16140800 & 0.09853300 \\ \mathrm{O} & 6.85499500 & 0.39604000 & 0.99093300 \\ \mathrm{O} & 1.09454500 & 3.77022800 & 4.14951000 \\ \mathrm{H} & 1.17237000 & 2.89060900 & 3.74609700 \\ \mathrm{H} & 1.45057500 & 4.35704600 & 3.47602300 \\ \mathrm{NU}-1000 & \mathrm{TS} 1 & & \\ \mathrm{C} & 5.63829400 & 0.42079600 & -2.05844700 \\ \mathrm{C} & -4.99310600 & 4.27690900 & -0.89151500 \\ \mathrm{C} & 4.50618900 & 1.24751300 & -2.00302000 \\ \mathrm{C} & -3.60179000 & 4.21864100 & -1.06240800 \\ \mathrm{C} & 4.64012000 & 2.60840500 & -2.31580000 \\ \mathrm{C} & -2.91688600 & 5.38120900 & -1.44642600 \\ \mathrm{C} & -3.87278200 & 3.12311800 & -2.70583000 \\ \mathrm{C} & 7.00838900 & 2.29490200 & -2.79810600 \\ \mathrm{C} & & & \\ \mathrm{C} & & & \end{array}$




$\begin{array}{lrrr}\mathrm{C} & 4.94555200 & -2.71881000 & 2.59410100 \\ \mathrm{C} & -5.63937500 & 1.22817100 & 3.66334400 \\ \mathrm{C} & 3.76597600 & -2.17953500 & 3.12850500 \\ \mathrm{C} & -4.30257300 & 0.89577200 & 3.93255700 \\ \mathrm{C} & 3.67697300 & -1.97068400 & 4.51230100 \\ \mathrm{C} & -3.86816700 & 0.85856100 & 5.26634200 \\ \mathrm{C} & 4.73284800 & -2.32292100 & 5.34742600 \\ \mathrm{C} & -4.75518900 & 1.12769100 & 6.30614400 \\ \mathrm{C} & 5.91131100 & -2.88960800 & 4.82405200 \\ \mathrm{C} & -6.10103800 & 1.45332500 & 6.04671600 \\ \mathrm{C} & 6.00305600 & -3.06262500 & 3.43007400 \\ \mathrm{C} & -6.52110000 & 1.50690800 & 4.70360800 \\ \mathrm{C} & -2.89005300 & 2.92177600 & -0.86448900 \\ \mathrm{C} & -4.40579700 & -0.57489900 & -3.35831400 \\ \mathrm{C} & -4.89533000 & -2.99187800 & 0.31650500 \\ \mathrm{C} & 1.23870400 & -5.12783000 & -0.15513700 \\ \mathrm{C} & 2.62998800 & -1.84573200 & 2.22173600 \\ \mathrm{C} & 3.17518200 & 0.68977600 & -1.63822600 \\ \mathrm{C} & -3.38945300 & 0.51917400 & 2.81602600 \\ \mathrm{C} & 1.73546900 & -2.57220100 & -4.01285200 \\ \mathrm{H} & -3.32077000 & -5.01340800 & -1.49522600 \\ \mathrm{H} & 5.54374400 & -0.63624300 & -1.80274800 \\ \mathrm{H} & -5.53428800 & 3.38140300 & -0.58192400 \\ \mathrm{H} & 3.76615800 & 3.25994800 & -2.26188500 \\ \mathrm{H} & -1.83239100 & 5.34735800 & -1.56999000 \\ \mathrm{H} & 5.95710100 & 4.18373800 & -2.95618100 \\ \mathrm{H} & -3.05892600 & 7.45558800 & -1.99698900 \\ \mathrm{H} & 7.74008400 & 0.27939300 & -2.49126500 \\ \mathrm{H} & -6.76815100 & 5.48693800 & -0.99139700 \\ \mathrm{H} & 5.02416500 & -2.87354300 & 1.51674000 \\ \mathrm{H} & -1.16220000 & -3.12509700 & 2.98433700 \\ \mathrm{H} & & & \\ \mathrm{H} & -5.98611000 & 1.25784800 & 2.62875300 \\ \mathrm{H} & 2.76602500 & -1.53920100 & 4.93238000 \\ \mathrm{H} & -2.83000800 & 0.60127900 & 5.48466600 \\ \mathrm{H} & 4.64385300 & -2.16145700 & 6.42464500 \\ \mathrm{H} & -4.40013700 & 1.08399500 & 7.33886200 \\ \mathrm{H} & 6.91299000 & -3.48758800 & 2.99876800 \\ \mathrm{H} & -7.55885500 & 1.76190300 & 4.47380800 \\ \mathrm{H} & -5.55509100 & 7.55143600 & -1.73880900 \\ \mathrm{H} & -6.79597400 & 1.66145900 & 6.86197400 \\ \mathrm{H} & 2.35913000 & 1.08238500 & -2.77765200 \\ \mathrm{H} & & -4.40015100 & -2.53749600 \\ \mathrm{H} & & & \end{array}$




\begin{tabular}{|c|c|c|c|}
\hline $\mathrm{H}$ & -1.25365200 & -5.09987000 & 2.09064500 \\
\hline $\mathrm{H}$ & 0.07117500 & -2.53524100 & 3.78550900 \\
\hline $\mathrm{H}$ & 0.00642600 & -3.98754100 & -2.33150700 \\
\hline $\mathrm{H}$ & -2.96310300 & -2.02738300 & 2.02484400 \\
\hline $\mathrm{H}$ & -2.75317500 & -3.35217000 & -4.38334900 \\
\hline $\mathrm{H}$ & -0.08694500 & -0.32035600 & -5.13587600 \\
\hline $\mathrm{H}$ & 0.87188300 & 2.30070700 & -3.39151000 \\
\hline $\mathrm{H}$ & -0.48313200 & 0.84343400 & -4.12455400 \\
\hline $\mathrm{O}$ & -2.35463300 & -1.91377800 & -1.23626100 \\
\hline $\mathrm{O}$ & -4.86368000 & -1.73697400 & 0.23918800 \\
\hline $\mathrm{O}$ & -3.89478600 & 0.46097800 & 1.65996200 \\
\hline $\mathrm{O}$ & -4.57278100 & -0.25280800 & -2.15425200 \\
\hline $\mathrm{O}$ & -3.38557800 & -1.09477700 & -3.87235500 \\
\hline $\mathrm{O}$ & -0.82383200 & 0.00597800 & -4.60136200 \\
\hline $\mathrm{O}$ & 0.56577400 & -2.22715600 & -4.30994100 \\
\hline $\mathrm{O}$ & 2.17812400 & 1.45769000 & -1.68501300 \\
\hline $\mathrm{O}$ & 2.23123400 & -2.66289200 & -2.86118800 \\
\hline $\mathrm{O}$ & 3.13499100 & -0.53787100 & -1.31949100 \\
\hline $\mathrm{O}$ & 1.93925900 & -4.14506100 & -0.50055400 \\
\hline $\mathrm{O}$ & 2.81541000 & -2.02143700 & 0.98390600 \\
\hline $\mathrm{O}$ & 1.56386200 & -1.40096900 & 2.73963500 \\
\hline $\mathrm{O}$ & 0.00419500 & -5.14482000 & 0.07553900 \\
\hline $\mathrm{O}$ & -0.00271000 & -2.26177900 & 0.56179900 \\
\hline $\mathrm{O}$ & -0.24478800 & -3.21088500 & -1.8162550 \\
\hline $\mathrm{O}$ & -2.00471600 & -3.43147100 & -3.78030900 \\
\hline $\mathrm{O}$ & -2.37479300 & -4.87135400 & -1.6367040 \\
\hline $\mathrm{O}$ & 0.29323700 & -0.70479200 & -1.91421000 \\
\hline $\mathrm{O}$ & -1.94579100 & 0.44875800 & -2.17843600 \\
\hline $\mathrm{O}$ & -1.63659000 & 2.90705400 & -0.98383200 \\
\hline $\mathrm{O}$ & -0.00673100 & 1.99052400 & -3.14062500 \\
\hline $\mathrm{O}$ & -3.61283100 & 1.90716900 & -0.62162200 \\
\hline $\mathrm{O}$ & -1.70431300 & -4.25408000 & 1.98035000 \\
\hline $\mathrm{O}$ & -0.80362100 & -2.29319200 & 3.45032300 \\
\hline $\mathrm{O}$ & -2.36324300 & -1.76090900 & 1.31714800 \\
\hline $\mathrm{O}$ & -3.92250700 & -3.78297900 & 0.24528500 \\
\hline $\mathrm{O}$ & -2.18807900 & 0.23759600 & 3.09805500 \\
\hline $\mathrm{O}$ & 1.12979700 & 0.03494900 & 0.41087500 \\
\hline $\mathrm{O}$ & -1.35521400 & 0.38528900 & 0.31329300 \\
\hline $\mathrm{Zr}$ & 1.51781000 & -1.96518900 & -0.8332990 \\
\hline $\mathrm{Zr}$ & -1.30203500 & -1.59594000 & -3.01677100 \\
\hline $\mathrm{Zr}$ & -1.60567900 & -3.47729000 & 0.02720300 \\
\hline $\mathrm{Zr}$ & -0.44800200 & -0.64427000 & 1.8624060 \\
\hline $\mathrm{Zr}$ & -3.22418400 & -0.26330700 & -0.3292950 \\
\hline $\mathrm{Zr}$ & -0.04752100 & 1.24279200 & -1.1577390 \\
\hline
\end{tabular}




$\begin{array}{lrrr}\mathrm{H} & 1.79940300 & -6.08676600 & -0.03740900 \\ \mathrm{H} & 2.43021400 & -2.79413200 & -4.86046600 \\ \mathrm{H} & -5.90768800 & -3.45544300 & 0.39958800 \\ \mathrm{H} & -5.26552900 & -0.38046400 & -4.04414800 \\ \mathrm{H} & 1.93366200 & 0.46699500 & 0.72297600 \\ \mathrm{O} & 0.25513700 & 1.09569700 & 2.95754200 \\ \mathrm{H} & -0.45087700 & 1.25650600 & 3.59957200 \\ \mathrm{O} & 0.75939000 & 2.71648600 & 0.23299800 \\ \mathrm{P} & 1.35384100 & 4.03889100 & 0.70256700 \\ \mathrm{O} & 2.03584900 & 4.64302500 & -0.67229100 \\ \mathrm{O} & 0.40427600 & 5.30843400 & 0.94904300 \\ \mathrm{O} & 2.82155900 & 4.20307700 & 1.46441800 \\ \mathrm{C} & 1.21016500 & 4.93311100 & -1.77770300 \\ \mathrm{H} & 1.83424500 & 4.90800000 & -2.68135500 \\ \mathrm{H} & 0.39290800 & 4.20359900 & -1.90433000 \\ \mathrm{H} & 0.77066100 & 5.93947900 & -1.68758800 \\ \mathrm{C} & -0.84483900 & 5.27084600 & 1.63287200 \\ \mathrm{H} & -1.43891000 & 6.09668400 & 1.22398900 \\ \mathrm{H} & -1.37249900 & 4.32482900 & 1.45313600 \\ \mathrm{H} & -0.70962400 & 5.41016200 & 2.71141600 \\ \mathrm{C} & 3.83210300 & 3.30210600 & 1.29689300 \\ \mathrm{C} & 5.07115700 & 3.77791900 & 0.86087900 \\ \mathrm{C} & 3.65879200 & 1.94772800 & 1.62176100 \\ \mathrm{C} & 6.14046300 & 2.90113000 & 0.73748200 \\ \mathrm{H} & 5.16808000 & 4.83541800 & 0.61234200 \\ \mathrm{C} & 4.71822000 & 1.06242500 & 1.46375100 \\ \mathrm{H} & 2.70498500 & 1.61918900 & 2.04382700 \\ \mathrm{C} & 5.94666500 & 1.55119200 & 1.02188200 \\ \mathrm{H} & 7.11908300 & 3.23697400 & 0.39406000 \\ \mathrm{H} & 4.61895800 & -0.00187600 & 1.68332800 \\ \mathrm{~N} & 7.05932500 & 0.61580900 & 0.83490900 \\ \mathrm{O} & 8.15625700 & 1.08546700 & 0.57774000 \\ \mathrm{O} & 6.81697300 & -0.57739200 & 0.93694100 \\ \mathrm{O} & 0.82424700 & 3.40729700 & 2.53435700 \\ \mathrm{H} & 1.47963800 & 3.67572000 & 3.18924500 \\ \mathrm{H} & 0.58662800 & 2.28265300 & 2.67053600\end{array}$

\begin{tabular}{|c|c|c|c|}
\hline \multicolumn{4}{|c|}{ NU-1000 INT 1} \\
\hline $\mathrm{C}$ & 5.63005400 & 0.33540800 & -2.06995800 \\
\hline $\mathrm{C}$ & -4.93817000 & 4.34855100 & -0.85945800 \\
\hline $\mathrm{C}$ & 4.51077400 & 1.17900200 & -2.00867000 \\
\hline $\mathrm{C}$ & -3.54841400 & 4.26973300 & -1.03474300 \\
\hline $\mathrm{C}$ & 4.66437100 & 2.53863600 & -2.31786200 \\
\hline
\end{tabular}




\begin{tabular}{|c|c|c|c|}
\hline $\mathrm{C}$ & -2.84712700 & 5.42293800 & -1.41742600 \\
\hline $\mathrm{C}$ & 5.90351700 & 3.03578600 & -2.71011900 \\
\hline $\mathrm{C}$ & -3.52316400 & 6.61715000 & -1.65194200 \\
\hline $\mathrm{C}$ & 7.02616600 & 2.19074900 & -2.80827200 \\
\hline $\mathrm{C}$ & -4.92190500 & 6.69956200 & -1.50510600 \\
\hline $\mathrm{C}$ & 6.86814100 & 0.83625100 & -2.45983700 \\
\hline $\mathrm{C}$ & -5.61108700 & 5.54513200 & -1.08637900 \\
\hline $\mathrm{C}$ & 4.90374400 & -2.80719700 & 2.57544000 \\
\hline $\mathrm{C}$ & -5.61692900 & 1.29639100 & 3.68837800 \\
\hline $\mathrm{C}$ & 3.73407600 & -2.25171900 & 3.11500100 \\
\hline $\mathrm{C}$ & -4.28451700 & 0.94298900 & 3.95255700 \\
\hline $\mathrm{C}$ & 3.65238600 & -2.04566800 & 4.49966800 \\
\hline $\mathrm{C}$ & -3.84673800 & 0.89523200 & 90200 \\
\hline $\mathrm{C}$ & 4.70529600 & -2.41633800 & 5.33054800 \\
\hline $\mathrm{C}$ & -4.72646800 & 1.17466300 & 6.32817600 \\
\hline $\mathrm{C}$ & 5.87346800 & -2.99924100 & 4.80194000 \\
\hline $\mathrm{C}$ & -6.06799900 & 1.52140800 & 6.07379100 \\
\hline $\mathrm{C}$ & 5.95841200 & -3.16947400 & 3.40718800 \\
\hline $\mathrm{C}$ & -6.49121600 & 1.58534600 & 4.73212700 \\
\hline $\mathrm{C}$ & -2.85581700 & 2.96165700 & -0.84279000 \\
\hline $\mathrm{C}$ & -4.43181100 & -0.50422200 & -3.34225100 \\
\hline $\mathrm{C}$ & -4.94691300 & -2.92445000 & 0.32693100 \\
\hline $\mathrm{C}$ & 1.15262100 & -5.15161500 & -0.16959000 \\
\hline $\mathrm{C}$ & 2.60056900 & -1.89805400 & 2.21266700 \\
\hline $\mathrm{C}$ & 3.17256700 & 0.64039600 & -1.64147600 \\
\hline $\mathrm{C}$ & -3.38055000 & 0.55593700 & 2.83215900 \\
\hline $\mathrm{C}$ & 1.67650800 & -2.59231900 & -4.02128100 \\
\hline $\mathrm{H}$ & 5.52026600 & -0.72083600 & -1.81707600 \\
\hline $\mathrm{H}$ & -5.49192900 & 3.46042300 & -0.55085400 \\
\hline $\mathrm{H}$ & 3.80054500 & 3.20317300 & -2.25938400 \\
\hline $\mathrm{H}$ & -1.76364500 & 5.37303900 & -1.54438000 \\
\hline $\mathrm{H}$ & 6.00314500 & 4.09574800 & -2.95761300 \\
\hline $\mathrm{H}$ & -2.95937100 & 7.50086000 & -1.96146700 \\
\hline $\mathrm{H}$ & 7.72815700 & 0.16348800 & -2.50956700 \\
\hline $\mathrm{H}$ & -6.69497100 & 5.58560800 & -0.95040200 \\
\hline $\mathrm{H}$ & 4.97678100 & -2.95989300 & 1.49739700 \\
\hline $\mathrm{H}$ & -5.96626700 & 1.33439600 & 2.65493600 \\
\hline $\mathrm{H}$ & 2.74933900 & -1.60169600 & 4.92377400 \\
\hline $\mathrm{H}$ & -2.81194800 & 0.62161200 & 5.49931800 \\
\hline $\mathrm{H}$ & 4.62197900 & -2.25675500 & 6.40850100 \\
\hline $\mathrm{H}$ & -4.36903200 & 1.12251900 & 7.35968000 \\
\hline $\mathrm{H}$ & 6.86051100 & -3.60687800 & 2.97187800 \\
\hline $\mathrm{H}$ & -7.52567100 & 1.85670700 & 4.50622700 \\
\hline & -5.45301400 & 7.63372100 & -1.6954320 \\
\hline
\end{tabular}




\begin{tabular}{|c|c|c|c|}
\hline $\mathrm{H}$ & 7.99503100 & 2.57954400 & -3.12632800 \\
\hline $\mathrm{H}$ & -6.75726200 & 1.73761000 & 6.89176200 \\
\hline $\mathrm{H}$ & 6.69652900 & -3.29559500 & 5.45448600 \\
\hline $\mathrm{H}$ & -2.36141300 & 1.11899100 & -2.74416500 \\
\hline $\mathrm{H}$ & -3.42093400 & -4.94788200 & -1.48194800 \\
\hline $\mathrm{H}$ & -2.38381200 & -4.35518200 & -2.53044400 \\
\hline $\mathrm{H}$ & -1.18440300 & -3.13549700 & 2.98127900 \\
\hline $\mathrm{H}$ & -1.32065400 & -5.10011900 & 2.04545200 \\
\hline $\mathrm{H}$ & 0.06273100 & -2.54492400 & 3.75850000 \\
\hline $\mathrm{H}$ & -0.06940500 & -3.98168100 & -2.34730400 \\
\hline $\mathrm{H}$ & -2.98616100 & -2.00775700 & 2.03911100 \\
\hline $\mathrm{H}$ & -2.81936000 & -3.27158100 & -4.37181900 \\
\hline $\mathrm{H}$ & -0.10975500 & -0.35915300 & -5.10641900 \\
\hline $\mathrm{H}$ & 0.86865300 & 2.30013700 & -3.37131400 \\
\hline $\mathrm{H}$ & -0.47862800 & 0.82689900 & -4.11200300 \\
\hline $\mathrm{O}$ & -2.39866600 & -1.88152800 & -1.22446600 \\
\hline $\mathrm{O}$ & -4.89658700 & -1.67197500 & 0.25438800 \\
\hline $\mathrm{O}$ & -3.89129900 & 0.49968300 & 1.68287500 \\
\hline $\mathrm{O}$ & -4.59416000 & -0.18340400 & -2.13792000 \\
\hline $\mathrm{O}$ & -3.42246000 & -1.03285400 & -3.86277600 \\
\hline $\mathrm{O}$ & -0.84214800 & 0.00750400 & -4.59449800 \\
\hline $\mathrm{O}$ & 0.51290900 & -2.23480700 & -4.31460200 \\
\hline $\mathrm{O}$ & 2.18936000 & 1.41918700 & -1.68970500 \\
\hline $\mathrm{O}$ & 2.17887300 & -2.69278800 & -2.87382700 \\
\hline $\mathrm{O}$ & 3.11652800 & -0.58766900 & -1.31797600 \\
\hline $\mathrm{O}$ & 1.86602500 & -4.18020400 & -0.51200200 \\
\hline $\mathrm{O}$ & 2.76964900 & -2.08852100 & 0.97911100 \\
\hline $\mathrm{O}$ & 1.54789700 & -1.41649200 & 2.73633400 \\
\hline $\mathrm{O}$ & -0.08191500 & -5.16015500 & 0.05813100 \\
\hline $\mathrm{O}$ & -0.04608500 & -2.28638600 & 0.56180800 \\
\hline $\mathrm{O}$ & -0.31110100 & -3.21176900 & -1.82292700 \\
\hline $\mathrm{O}$ & -2.06632800 & -3.37839500 & -3.78308200 \\
\hline $\mathrm{O}$ & -2.47518100 & -4.84004200 & -1.64334900 \\
\hline $\mathrm{O}$ & 0.26939200 & -0.70956000 & -1.89491600 \\
\hline $\mathrm{O}$ & -1.95118400 & 0.47825800 & -2.15483500 \\
\hline $\mathrm{O}$ & -1.60744200 & 2.92901400 & -0.97090300 \\
\hline $\mathrm{O}$ & -0.00358100 & 1.97688000 & -3.12350000 \\
\hline $\mathrm{O}$ & -3.59294100 & 1.95910600 & -0.58866800 \\
\hline $\mathrm{O}$ & -1.78137000 & -4.26009500 & 1.95540100 \\
\hline $\mathrm{O}$ & -0.82319000 & -2.31381100 & 3.44845700 \\
\hline $\mathrm{O}$ & -2.39253000 & -1.75285500 & 1.32592200 \\
\hline $\mathrm{O}$ & -3.99237700 & -3.73574700 & 0.25315800 \\
\hline $\mathrm{O}$ & -2.17709400 & 0.26106700 & 3.10762700 \\
\hline $\mathrm{O}$ & 1.12140000 & 0.01473100 & 0.43001200 \\
\hline
\end{tabular}




\begin{tabular}{|c|c|c|c|}
\hline $\mathrm{O}$ & -1.35228100 & 0.39542700 & 0.33905000 \\
\hline $\mathrm{Zr}$ & 1.47859400 & -1.98393100 & -0.85404700 \\
\hline $\mathrm{Zr}$ & -1.34412800 & -1.56686500 & -3.00204100 \\
\hline $\mathrm{Zr}$ & -1.67173500 & -3.47488500 & 0.01734500 \\
\hline $\mathrm{Zr}$ & -0.46633700 & -0.69579400 & 1.82980700 \\
\hline $\mathrm{Zr}$ & -3.23214900 & -0.21746900 & -0.32190300 \\
\hline $\mathrm{Zr}$ & -0.02580900 & 1.27328800 & -1.13913800 \\
\hline $\mathrm{H}$ & 1.69907800 & -6.11927800 & -0.05637800 \\
\hline $\mathrm{H}$ & 2.36527400 & -2.82221700 & -4.87164700 \\
\hline $\mathrm{H}$ & -5.96592400 & -3.37288000 & 0.41172600 \\
\hline $\mathrm{H}$ & -5.29054500 & -0.29475000 & -4.02489900 \\
\hline $\mathrm{H}$ & 1.94812000 & 0.40711900 & 0.72795700 \\
\hline $\mathrm{O}$ & 0.23012300 & 1.11634600 & 3.03184700 \\
\hline $\mathrm{H}$ & -0.58021100 & 1.35214300 & 3.50790300 \\
\hline $\mathrm{O}$ & 0.78382600 & 2.70115200 & 0.23555400 \\
\hline $\mathrm{P}$ & 1.40729100 & 3.99933900 & 0.74544800 \\
\hline $\mathrm{O}$ & 2.10948200 & 4.50428900 & -0.67231500 \\
\hline $\mathrm{O}$ & 0.51007700 & 5.32762200 & 0.80158400 \\
\hline $\mathrm{O}$ & 2.94158100 & 4.19415900 & 1.39242900 \\
\hline $\mathrm{C}$ & 1.29050300 & 4.79982900 & -1.77721800 \\
\hline $\mathrm{H}$ & 1.87858000 & 4.64634200 & -2.68411600 \\
\hline $\mathrm{H}$ & 0.40139500 & 4.16468300 & -1.83435800 \\
\hline $\mathrm{H}$ & 0.96194500 & 5.84268200 & -1.75048100 \\
\hline $\mathrm{C}$ & -0.68174400 & 5.43930600 & 1.57043800 \\
\hline $\mathrm{H}$ & -1.23451700 & 6.27672600 & 1.14925800 \\
\hline $\mathrm{H}$ & -1.28992300 & 4.53723800 & 1.49760300 \\
\hline $\mathrm{H}$ & -0.46462800 & 5.63807100 & 2.61874900 \\
\hline $\mathrm{C}$ & 3.93205300 & 3.29027000 & 1.24536100 \\
\hline $\mathrm{C}$ & 5.18700200 & 3.75254100 & 0.85585000 \\
\hline $\mathrm{C}$ & 3.74893400 & 1.93711100 & 1.55130100 \\
\hline $\mathrm{C}$ & 6.24559000 & 2.87371200 & 0.75720500 \\
\hline $\mathrm{H}$ & 5.30155100 & 4.80128600 & 0.61964100 \\
\hline $\mathrm{C}$ & 4.80062300 & 1.05107600 & 1.42883100 \\
\hline $\mathrm{H}$ & 2.78963400 & 1.61396000 & 1.93784500 \\
\hline $\mathrm{C}$ & 6.04028500 & 1.53024500 & 1.03163800 \\
\hline $\mathrm{H}$ & 7.22681300 & 3.20197800 & 0.44566600 \\
\hline $\mathrm{H}$ & 4.68709100 & -0.00315600 & 1.64323900 \\
\hline $\mathrm{N}$ & 7.15319700 & 0.59480000 & 0.88680800 \\
\hline $\mathrm{O}$ & 8.25708200 & 1.05728200 & 0.63842500 \\
\hline $\mathrm{O}$ & 6.91265700 & -0.59799500 & 1.01432900 \\
\hline $\mathrm{O}$ & 0.94086700 & 3.52940300 & 2.38015400 \\
\hline $\mathrm{H}$ & 1.51145100 & 3.96960200 & 3.01783100 \\
\hline $\mathrm{H}$ & 0.57455400 & 1.99122600 & 2.67881200 \\
\hline
\end{tabular}




$\begin{array}{lrrr}\text { NU-1000 TS Berry } & & \\ \mathrm{C} & 5.64731900 & 0.37331200 & -2.04103000 \\ \mathrm{C} & -4.97698400 & 4.27679100 & -0.97096600 \\ \mathrm{C} & 4.51757100 & 1.20414600 & -2.00128700 \\ \mathrm{C} & -3.58512300 & 4.21204900 & -1.13494800 \\ \mathrm{C} & 4.65712100 & 2.56046100 & -2.33103100 \\ \mathrm{C} & -2.89491500 & 5.36744700 & -1.53092800 \\ \mathrm{C} & 5.89307900 & 3.06630200 & -2.72223200 \\ \mathrm{C} & -3.58348400 & 6.54950400 & -1.78934700 \\ \mathrm{C} & 7.02648600 & 2.23348100 & -2.79878700 \\ \mathrm{C} & -4.98416200 & 6.61727900 & -1.65393200 \\ \mathrm{C} & 6.88215600 & 0.88287000 & -2.42992000 \\ \mathrm{C} & -5.66251700 & 5.46137500 & -1.22180700 \\ \mathrm{C} & 4.92442400 & -2.70371400 & 2.64859100 \\ \mathrm{C} & -5.65269600 & 1.28921800 & 3.62000600 \\ \mathrm{C} & 3.74421200 & -2.15395700 & 3.17077000 \\ \mathrm{C} & -4.31813000 & 0.95622900 & 3.89939200 \\ \mathrm{C} & 3.64980900 & -1.92695300 & 4.55134600 \\ \mathrm{C} & -3.88968100 & 0.93494100 & 5.23545000 \\ \mathrm{C} & 4.70091500 & -2.27159600 & 5.39560600 \\ \mathrm{H} & -5.99480700 & 1.30657400 & 2.58359800 \\ \mathrm{H} & 2.73838400 & -1.48728000 & 4.96178900 \\ \mathrm{H} & -4.78039800 & 1.22021800 & 6.26776200 \\ \mathrm{C} & 5.87987900 & -2.84861800 & 4.88480600 \\ \mathrm{H} & -6.12407600 & 1.54659600 & 5.99820900 \\ \mathrm{H} & -3.02806400 & 7.43495700 & -2.10886800 \\ \mathrm{C} & 5.97718000 & -3.03993200 & 3.49359800 \\ \mathrm{C} & -6.53808900 & 1.58409100 & 4.65268000 \\ \mathrm{C} & -2.87831400 & 2.91567500 & -0.91714900 \\ \mathrm{C} & -4.39405200 & -0.60829600 & -3.37225400 \\ \mathrm{C} & -4.90720600 & -2.97602900 & 0.33129000 \\ \mathrm{C} & 1.22213000 & -5.13670900 & -0.08562900 \\ \mathrm{C} & 2.61325000 & -1.82842600 & 2.25475700 \\ \mathrm{C} & 3.18324400 & 0.65525800 & -1.63519700 \\ \mathrm{C} & -3.40131500 & 0.56242200 & 2.79186100 \\ \mathrm{C} & 1.74374900 & -2.63272700 & -3.97381200 \\ \mathrm{H} & 5.54834900 & -0.68003700 & -1.77211500 \\ \mathrm{H} & -5.52231100 & 3.38702800 & -0.65222300 \\ \mathrm{H} & 3.78497100 & 3.21532500 & -2.28939800 \\ \mathrm{H} & -1.81000300 & 5.32867400 & -1.64925500 \\ \mathrm{H} & 5.98180600 & 4.12333100 & -2.98589200 \\ \mathrm{H} & 5.007266500 & -2.87261100 & 1.57367800 \\ \mathrm{H} & & & \\ \mathrm{H} & & & \end{array}$




\begin{tabular}{|c|c|c|c|}
\hline $\mathrm{H}$ & -2.85329600 & 0.67732100 & 5.46166100 \\
\hline $\mathrm{H}$ & 4.60771300 & -2.09594000 & 6.47024500 \\
\hline $\mathrm{H}$ & -4.43000600 & 1.18879400 & 7.30251600 \\
\hline $\mathrm{H}$ & 6.88766100 & -3.47322700 & 3.07184100 \\
\hline $\mathrm{H}$ & -7.57402600 & 1.83927400 & 4.41502500 \\
\hline $\mathrm{H}$ & -5.52502300 & 7.54179300 & -1.86297100 \\
\hline $\mathrm{H}$ & 7.99294200 & 2.62889100 & -3.11601700 \\
\hline $\mathrm{H}$ & -6.82191900 & 1.76738800 & 6.80763500 \\
\hline $\mathrm{H}$ & 6.70158600 & -3.12457700 & 5.54791600 \\
\hline $\mathrm{H}$ & -2.34133600 & 1.05677100 & -2.78821700 \\
\hline $\mathrm{H}$ & -3.33541600 & -5.03764800 & -1.48972200 \\
\hline $\mathrm{H}$ & -2.28880000 & -4.41509600 & -2.5203920 \\
\hline $\mathrm{H}$ & -1.19022000 & -3.11892200 & 3.00590400 \\
\hline $\mathrm{H}$ & -1.28209300 & -5.08701200 & 2.12917600 \\
\hline $\mathrm{H}$ & 0.04239300 & -2.52467600 & 3.81196800 \\
\hline $\mathrm{H}$ & 0.00286500 & -4.01448900 & -2.29321100 \\
\hline $\mathrm{H}$ & -2.97612700 & -2.01414100 & 2.04054700 \\
\hline $\mathrm{H}$ & -2.73635500 & -3.38057000 & -4.36123100 \\
\hline $\mathrm{H}$ & -0.05985100 & -0.35584800 & -5.12594100 \\
\hline $\mathrm{H}$ & 0.89380900 & 2.28130300 & -3.39616000 \\
\hline $\mathrm{H}$ & -0.44980700 & 0.81135500 & -4.11213800 \\
\hline $\mathrm{O}$ & -2.35476900 & -1.92579100 & -1.22136900 \\
\hline $\mathrm{O}$ & -4.86938300 & -1.72286500 & 0.24174000 \\
\hline $\mathrm{O}$ & -3.90260800 & 0.47967500 & 1.63827100 \\
\hline $\mathrm{O}$ & -4.56873700 & -0.27676800 & -2.17116600 \\
\hline $\mathrm{O}$ & -3.36808500 & -1.12707600 & -3.8744780 \\
\hline $\mathrm{O}$ & -0.79319600 & -0.02914900 & -4.58716400 \\
\hline $\mathrm{O}$ & 0.58023000 & -2.27480300 & -4.27759600 \\
\hline $\mathrm{O}$ & 2.19011600 & 1.42786500 & -1.67342400 \\
\hline $\mathrm{O}$ & 2.23324700 & -2.71907200 & -2.81857100 \\
\hline $\mathrm{O}$ & 3.13421900 & -0.57413700 & -1.31404500 \\
\hline $\mathrm{O}$ & 1.92522100 & -4.15948600 & -0.43787800 \\
\hline $\mathrm{O}$ & 2.79404000 & -2.03950700 & 1.02524500 \\
\hline $\mathrm{O}$ & 1.54689400 & -1.35416100 & 2.76163300 \\
\hline $\mathrm{O}$ & -0.01633200 & -5.15193300 & 0.13005300 \\
\hline $\mathrm{O}$ & -0.02068900 & -2.27867400 & 0.58843100 \\
\hline $\mathrm{O}$ & -0.24895900 & -3.23544700 & -1.78169800 \\
\hline $\mathrm{O}$ & -1.99435300 & -3.46020900 & -3.7502730 \\
\hline $\mathrm{O}$ & -2.38905100 & -4.88598400 & -1.6163990 \\
\hline $\mathrm{O}$ & 0.29598300 & -0.72848200 & -1.88458200 \\
\hline $\mathrm{O}$ & -1.93400100 & 0.42797400 & -2.17966800 \\
\hline $\mathrm{O}$ & -1.62654900 & 2.89678300 & -1.0303890 \\
\hline $\mathrm{O}$ & 0.02051100 & 1.95850700 & -3.14280500 \\
\hline $\mathrm{O}$ & -3.60439400 & 1.90454300 & -0.6575880 \\
\hline
\end{tabular}




\begin{tabular}{|c|c|c|c|}
\hline $\mathrm{O}$ & -1.73272000 & -4.24201900 & 2.01136100 \\
\hline $\mathrm{O}$ & -0.82640200 & -2.28093500 & 3.46287900 \\
\hline $\mathrm{O}$ & -2.37979300 & -1.75972000 & 1.32533100 \\
\hline $\mathrm{O}$ & -3.93911800 & -3.77467000 & 0.26497300 \\
\hline $\mathrm{O}$ & -2.19435100 & 0.28255300 & 3.07791500 \\
\hline $\mathrm{O}$ & 1.12704300 & 0.01210400 & 0.42901400 \\
\hline $\mathrm{O}$ & -1.35827500 & 0.37673300 & 0.31595800 \\
\hline $\mathrm{Zr}$ & 1.51930900 & -1.97989500 & -0.81486000 \\
\hline $\mathrm{Zr}$ & -1.28590300 & -1.61636600 & -2.99916500 \\
\hline $\mathrm{Zr}$ & -1.63191500 & -3.49442200 & 0.04337000 \\
\hline $\mathrm{Zr}$ & -0.46639400 & -0.67768600 & 1.83364100 \\
\hline $\mathrm{Zr}$ & -3.22827000 & -0.25891400 & -0.34767700 \\
\hline $\mathrm{Zr}$ & -0.02993300 & 1.25823600 & -1.14383600 \\
\hline $\mathrm{H}$ & 1.77931000 & -6.09575900 & 0.04695300 \\
\hline $\mathrm{H}$ & 2.44150000 & -2.86773300 & -4.81541200 \\
\hline $\mathrm{H}$ & -5.92136000 & -3.43537400 & 0.41588600 \\
\hline $\mathrm{H}$ & -5.25016200 & -0.42011200 & -4.06433200 \\
\hline $\mathrm{H}$ & 1.91654500 & 0.47027500 & 0.74730200 \\
\hline $\mathrm{O}$ & 0.20633000 & 1.20217000 & 2.98315100 \\
\hline $\mathrm{H}$ & -0.56903200 & 1.36831000 & 3.54414100 \\
\hline $\mathrm{O}$ & 0.70856300 & 2.62704600 & 0.32595300 \\
\hline $\mathrm{P}$ & 1.20707100 & 3.99823100 & 0.85568000 \\
\hline $\mathrm{O}$ & 2.21784600 & 4.45613700 & -0.40039400 \\
\hline $\mathrm{O}$ & 0.32037900 & 5.35117200 & 0.56955900 \\
\hline $\mathrm{O}$ & 2.61408100 & 3.72496700 & 1.78631100 \\
\hline $\mathrm{C}$ & 1.64955000 & 4.80977400 & -1.63499500 \\
\hline $\mathrm{H}$ & 2.41075100 & 4.66535100 & -2.41606800 \\
\hline $\mathrm{H}$ & 0.77444200 & 4.18664400 & -1.89495600 \\
\hline $\mathrm{H}$ & 1.33169400 & 5.86364500 & -1.65492500 \\
\hline $\mathrm{C}$ & -0.89728200 & 5.68768900 & 1.20794600 \\
\hline $\mathrm{H}$ & -1.31044700 & 6.53842700 & 0.65058400 \\
\hline $\mathrm{H}$ & -1.61915300 & 4.85945400 & 1.18038600 \\
\hline $\mathrm{H}$ & -0.74552700 & 5.98755600 & 2.25247600 \\
\hline $\mathrm{C}$ & 3.72500400 & 3.04992300 & 1.41570400 \\
\hline $\mathrm{C}$ & 4.84447000 & 3.69755300 & 0.88065900 \\
\hline $\mathrm{C}$ & 3.78410600 & 1.67783800 & 1.71667500 \\
\hline $\mathrm{C}$ & 6.01825900 & 2.98582700 & 0.67123500 \\
\hline $\mathrm{H}$ & 4.77359300 & 4.75944000 & 0.64432600 \\
\hline $\mathrm{C}$ & 4.94273300 & 0.95378600 & 1.47305400 \\
\hline $\mathrm{H}$ & 2.92909400 & 1.21376100 & 2.21859600 \\
\hline $\mathrm{C}$ & 6.05330100 & 1.62121000 & 0.95931500 \\
\hline $\mathrm{H}$ & 6.91219200 & 3.46450400 & 0.27056500 \\
\hline $\mathrm{H}$ & 5.01033400 & -0.11307900 & 1.69038600 \\
\hline $\mathrm{N}$ & 7.28223700 & 0.86583100 & 0.71694700 \\
\hline
\end{tabular}




$\begin{array}{lrrr}\mathrm{O} & 8.28115700 & 1.49177000 & 0.39657100 \\ \mathrm{O} & 7.23374100 & -0.35017000 & 0.84015400 \\ \mathrm{O} & 0.41231300 & 3.79428700 & 2.40051100 \\ \mathrm{H} & 1.00609400 & 4.10081800 & 3.09765700 \\ \mathrm{H} & 0.36925900 & 2.10035100 & 2.57398500\end{array}$

NU-1000 INT Berry

$\begin{array}{llll}\text { C } & 5.28580900 & -0.24112900 & -2.66872700\end{array}$

$\begin{array}{lllll}\text { C } & -4.78240000 & 4.70038600 & -0.80578000\end{array}$

$\begin{array}{lllll}\text { C } & 4.24435500 & 0.69367900 & -2.57022000\end{array}$

C $\quad \begin{array}{llll}\text { C } & -3.42628300 & 4.49574700 & -1.10146100\end{array}$

$\begin{array}{lllll}\text { C } & 4.46859200 & 2.01120000 & -2.99642800\end{array}$

C $\quad-2.68120400 \quad 5.55796500 \quad-1.63501500$

$\begin{array}{llll}\text { C } & 5.69726200 & 2.37637900 & -3.53820300\end{array}$

$\begin{array}{lllll}\text { C } & -3.28539400 & 6.78432000 & -1.89802900\end{array}$

$\begin{array}{llll}\text { C } & 6.73834800 & 1.43728600 & -3.67350600\end{array}$

C $\quad \begin{array}{lllll}\text { C } & -4.65293900 & 6.99128400 & -1.63004300\end{array}$

$\begin{array}{lllll}\text { C } & 6.51393800 & 0.12797600 & -3.20832800\end{array}$

C $\quad \begin{array}{llll}\text { C } & -5.38257300 & 5.92937000 & -1.06172300\end{array}$

$\begin{array}{llll}\mathrm{C} & 4.78324500 & -2.98141000 & 2.25280800\end{array}$

$\begin{array}{llll}\text { C } & -5.24052400 & 2.03930800 & 4.00753400\end{array}$

$\begin{array}{lllll}\text { C } & 3.71691300 & -2.29550400 & 2.85303600\end{array}$

$\begin{array}{llll}\text { C } & -3.91896600 & 1.59708000 & 4.17463900\end{array}$

$\begin{array}{llll}\text { C } & 3.78664500 & -1.98724700 & 4.21925200\end{array}$

$\begin{array}{lllll}\text { C } & -3.35788400 & 1.60672700 & 5.46078400\end{array}$

$\begin{array}{llll}\text { C } & 4.88492500 & -2.38413000 & 4.97598500\end{array}$

$\begin{array}{lllll}\text { C } & -4.10797100 & 2.02958000 & 6.55577000\end{array}$

$\begin{array}{lllll}\text { C } & 5.94878500 & -3.09641300 & 4.38888800\end{array}$

$\begin{array}{llll}\text { C } & -5.43812100 & 2.46662200 & 6.39974100\end{array}$

$\begin{array}{llll}\text { C } & 5.88398400 & -3.37003300 & 3.00959100\end{array}$

$\begin{array}{llll}\text { C } & -5.98444800 & 2.47121700 & 5.10175500\end{array}$

$\begin{array}{lllll}\text { C } & & -2.81836400 & 3.15186000 & -0.87341900\end{array}$

$\begin{array}{lllll}\text { C } & -4.88673400 & -0.33907700 & -2.94189500\end{array}$

$\begin{array}{llll}\text { C } & -5.22090500 & -2.44669500 & 0.93521800\end{array}$

$\begin{array}{lllll}\mathrm{C} & 0.61648100 & -5.19608000 & 0.05378100\end{array}$

$\begin{array}{llll}\mathrm{C} & 2.53039100 & -1.91390300 & 2.03409100\end{array}$

$\begin{array}{lllll}\text { C } & 2.91181800 & 0.29366300 & -2.04101500\end{array}$

C $\quad \begin{array}{llll}\text { C } & -3.16044100 & 1.05985700 & 3.00918300\end{array}$

C $\quad 0.95196800 \quad-2.96367000 \quad-4.01608000$

$\mathrm{H} \quad \begin{array}{llll}\mathrm{C} & 5.12231100 & -1.26455300 & -2.32590800\end{array}$

$\mathrm{H} \quad-5.36842400 \quad 3.88442200 \quad-0.37982800$

$\mathrm{H} \quad 3.66693400 \quad 2.74683400 \quad-2.91034900$

$\mathrm{H} \quad \begin{array}{llll}3.62213300 & 5.41058300 & -1.85679500\end{array}$

$\mathrm{H} \quad \begin{array}{llll}\mathrm{H} & 5.85148300 & 3.40471000 & -3.87502700\end{array}$ 


$\begin{array}{lrrr}\mathrm{H} & -2.68983500 & 7.59500500 & -2.32547700 \\ \mathrm{H} & 7.31205300 & -0.61491400 & -3.28473700 \\ \mathrm{H} & -6.44186900 & 6.06804800 & -0.83022000 \\ \mathrm{H} & 4.73889900 & -3.21449900 & 1.18766500 \\ \mathrm{H} & -5.68535600 & 2.03358400 & 3.01075200 \\ \mathrm{H} & 2.96524900 & -1.44213900 & 4.68897400 \\ \mathrm{H} & -2.33055700 & 1.26476300 & 5.59967800 \\ \mathrm{H} & 4.91957700 & -2.14335700 & 6.04145000 \\ \mathrm{H} & -3.65635300 & 2.02044800 & 7.55097600 \\ \mathrm{H} & 6.70383600 & -3.90931500 & 2.52832600 \\ \mathrm{H} & -7.01273500 & 2.81010600 & 4.95160500 \\ \mathrm{H} & -5.12850100 & 7.95029300 & -1.84240100 \\ \mathrm{H} & 7.69791800 & 1.72210300 & -4.10839100 \\ \mathrm{H} & -6.02595800 & 2.79525100 & 7.25843400 \\ \mathrm{H} & 6.80711300 & -3.41300700 & 4.98396100 \\ \mathrm{H} & -2.63823300 & 1.15776700 & -2.66827000 \\ \mathrm{H} & -4.03866700 & -4.76165100 & -0.90544600 \\ \mathrm{H} & -3.05662100 & -4.28451200 & -2.07002600 \\ \mathrm{H} & -1.28357900 & -2.81705300 & 3.23053100 \\ \mathrm{H} & -1.63281300 & -4.80081800 & 2.48609300 \\ \mathrm{H} & 0.06315200 & -2.29993100 & 3.90147100 \\ \mathrm{H} & -0.71960900 & -4.08561100 & -2.09234000 \\ \mathrm{H} & -3.05344400 & -1.58238000 & 2.37721000 \\ \mathrm{O} & -3.60362900 & -3.30845300 & -3.91404200 \\ \mathrm{O} & -0.29541400 & -2.20915500 & 0.66306400 \\ \mathrm{H} & -0.74790800 & -0.59456400 & -5.12609200 \\ \mathrm{H} & 0.60795500 & 2.04499600 & -3.65888800 \\ \mathrm{H} & -0.92851200 & 0.65769100 & -4.15836800 \\ \mathrm{O} & -2.75582700 & -1.72493700 & -0.92414400 \\ \mathrm{O} & -5.08012300 & -1.21045700 & 0.76120000 \\ \mathrm{O} & -3.79109200 & 0.94971600 & 1.92248200 \\ \mathrm{O} & -4.90427400 & 0.07069700 & -1.75293400 \\ \mathrm{O} & -3.96888500 & -0.97922700 & -3.51030600 \\ \mathrm{O} & -1.39036900 & -0.17096500 & -4.54064300 \\ \mathrm{O} & -0.20281700 & -2.51945400 & -4.22656100 \\ \mathrm{O} & 1.98630600 & 1.15003600 & -2.03293800 \\ \mathrm{O} & 1.54905400 & -3.02905800 & -2.91128000 \\ \mathrm{O} & 2.78583000 & -0.90835300 & -1.64631900 \\ \mathrm{O} & 1.36089800 & -4.31018600 & -0.43064300 \\ \mathrm{O} & 1.569075900 & -2.22502900 & 0.81227600 \\ \mathrm{O} & -3.597500 & -3.42458700 & -3.37514500\end{array}$




\begin{tabular}{|c|c|c|c|}
\hline $\mathrm{O}$ & -3.10129400 & -4.69445800 & -1.13199600 \\
\hline $\mathrm{O}$ & -0.10309400 & -0.82538300 & -1.92159500 \\
\hline $\mathrm{O}$ & -2.23279000 & 0.51973700 & -2.06784500 \\
\hline $\mathrm{O}$ & -1.59312200 & 2.99827700 & -1.10811000 \\
\hline $\mathrm{O}$ & -0.25704400 & 1.80803600 & -3.30298100 \\
\hline $\mathrm{O}$ & -3.60222700 & 2.22979000 & -0.47710200 \\
\hline $\mathrm{O}$ & -2.01879400 & -3.92498000 & 2.36337300 \\
\hline $\mathrm{O}$ & -0.80250800 & -1.99279100 & 3.59841600 \\
\hline $\mathrm{O}$ & -2.50842000 & -1.42814200 & 1.59539600 \\
\hline $\mathrm{O}$ & -4.34059600 & -3.33740000 & 0.82293500 \\
\hline $\mathrm{O}$ & -1.94841700 & 0.70741400 & 3.17882600 \\
\hline $\mathrm{O}$ & 1.03354800 & -0.04341900 & 0.24950100 \\
\hline $\mathrm{O}$ & -1.40793700 & 0.55819500 & 0.36294200 \\
\hline $\mathrm{Zr}$ & 1.11522500 & -2.12253900 & -0.89493800 \\
\hline $\mathrm{Zr}$ & -1.86507200 & -1.62101100 & -2.81885000 \\
\hline $\mathrm{Zr}$ & -2.05997900 & -3.29431500 & 0.35287200 \\
\hline $\mathrm{Zr}$ & -0.46324300 & -0.52689200 & 1.84760200 \\
\hline $\mathrm{Zr}$ & -3.38583100 & 0.06268000 & -0.07375200 \\
\hline $\mathrm{Zr}$ & -0.16744100 & 1.20277100 & -1.28323300 \\
\hline $\mathrm{H}$ & 1.09722200 & -6.19504300 & 0.19076400 \\
\hline $\mathrm{H}$ & 1.53511700 & -3.30813600 & -4.90582900 \\
\hline $\mathrm{H}$ & -6.25751400 & -2.80297500 & 1.14775200 \\
\hline $\mathrm{H}$ & -5.78992600 & -0.10803400 & -3.55685400 \\
\hline $\mathrm{H}$ & 1.86282600 & 0.43570600 & 0.46151000 \\
\hline $\mathrm{O}$ & 0.46466800 & 1.48326800 & 2.50861000 \\
\hline $\mathrm{H}$ & -0.24116600 & 1.94326700 & 2.98913500 \\
\hline $\mathrm{O}$ & 0.83400700 & 2.63594700 & 0.07720100 \\
\hline $\mathrm{P}$ & 2.08910500 & 3.52643900 & 0.33831400 \\
\hline $\mathrm{O}$ & 3.24915100 & 3.77874100 & -0.75430200 \\
\hline $\mathrm{O}$ & 1.40919400 & 5.01395400 & 0.05997800 \\
\hline $\mathrm{O}$ & 3.01555700 & 1.90645700 & 0.66162000 \\
\hline $\mathrm{C}$ & 3.50429900 & 5.01007000 & -1.41290900 \\
\hline $\mathrm{H}$ & 4.44888000 & 4.86628300 & -1.95338400 \\
\hline $\mathrm{H}$ & 2.70484300 & 5.26308300 & -2.12132900 \\
\hline $\mathrm{H}$ & 3.61500500 & 5.83741000 & -0.70125700 \\
\hline $\mathrm{C}$ & 0.22933500 & 5.38862400 & 0.72981900 \\
\hline $\mathrm{H}$ & -0.06563300 & 6.37867700 & 0.35893600 \\
\hline $\mathrm{H}$ & -0.59211100 & 4.68362300 & 0.52526900 \\
\hline $\mathrm{H}$ & 0.38538500 & 5.46252900 & 1.81808800 \\
\hline $\mathrm{C}$ & 4.32828800 & 1.74208700 & 0.82923200 \\
\hline $\mathrm{C}$ & 5.22798000 & 2.79445100 & 1.11134500 \\
\hline $\mathrm{C}$ & 4.83625900 & 0.42733000 & 0.74511900 \\
\hline $\mathrm{C}$ & 6.57933600 & 2.53644200 & 1.28261400 \\
\hline $\mathrm{H}$ & 4.86335300 & 3.81819300 & 1.19618700 \\
\hline
\end{tabular}




$\begin{array}{lrrr}\mathrm{C} & 6.18330400 & 0.17003500 & 0.92154600 \\ \mathrm{H} & 4.15045100 & -0.38845400 & 0.50518100 \\ \mathrm{C} & 7.05428200 & 1.22841300 & 1.18437500 \\ \mathrm{H} & 7.28592100 & 3.33859300 & 1.49701300 \\ \mathrm{H} & 6.58308100 & -0.84157800 & 0.84563400 \\ \mathrm{~N} & 8.47529400 & 0.96196200 & 1.35900700 \\ \mathrm{O} & 9.21275800 & 1.91452300 & 1.57546600 \\ \mathrm{O} & 8.85126900 & -0.20045800 & 1.27886600 \\ \mathrm{O} & 2.35424800 & 3.88580700 & 1.91187600 \\ \mathrm{H} & 2.72136800 & 3.11866500 & 2.37328700 \\ \mathrm{H} & 0.58127100 & 1.98423100 & 1.65835900\end{array}$

NU-1000 TS 2

$\begin{array}{lrrr}\mathrm{C} & 5.38056000 & -0.21315700 & -2.43785000 \\ \mathrm{C} & -4.74696600 & 4.71635100 & -0.89151300 \\ \mathrm{C} & 4.33693300 & 0.72166700 & -2.36615500 \\ \mathrm{C} & -3.38074700 & 4.51390100 & -1.13813700 \\ \mathrm{C} & 4.57840300 & 2.04462200 & -2.76525100 \\ \mathrm{C} & -2.61494900 & 5.58232700 & -1.62818500 \\ \mathrm{C} & 5.82714300 & 2.41551000 & -3.25473700 \\ \mathrm{C} & -3.20760000 & 6.81304000 & -1.89703100 \\ \mathrm{C} & 6.87180200 & 1.47691000 & -3.36327000 \\ \mathrm{C} & -4.58418100 & 7.01821100 & -1.67848200 \\ \mathrm{C} & 6.62868100 & 0.16162600 & -2.92513000 \\ \mathrm{C} & -5.33587000 & 5.94959000 & -1.15310400 \\ \mathrm{C} & 4.68844600 & -3.02032700 & 2.42260300 \\ \mathrm{C} & -5.39047900 & 1.98980500 & 3.86359400 \\ \mathrm{C} & 3.60062400 & -2.34126300 & 2.99121500 \\ \mathrm{C} & -4.07663700 & 1.54345800 & 4.07477400 \\ \mathrm{C} & 3.61852400 & -2.05197100 & 4.36322900 \\ \mathrm{C} & -3.56495500 & 1.53458200 & 5.38136500 \\ \mathrm{C} & 4.68682600 & -2.46079400 & 5.15567700 \\ \mathrm{C} & -4.35584300 & 1.94335100 & 6.45273400 \\ \mathrm{C} & 5.77165500 & -3.16640900 & 4.59972500 \\ \mathrm{C} & -5.67869800 & 2.38437300 & 6.25222200 \\ \mathrm{C} & 5.75921100 & -3.42089400 & 3.21530800 \\ \mathrm{C} & -6.17517200 & 2.40763100 & 4.93455900 \\ \mathrm{C} & -2.78314100 & 3.16614500 & -0.90571900 \\ \mathrm{C} & -4.77427200 & -0.29302900 & -3.09955200 \\ \mathrm{C} & -5.25776800 & -2.45343300 & 0.73249900 \\ \mathrm{C} & 0.60654100 & -5.19860600 & 0.03606500 \\ \mathrm{C} & 2.44650000 & -1.94673900 & 2.13303400 \\ \mathrm{C} & 2.98484900 & 0.31626600 & -1.89366500 \\ \mathrm{C} & -3.27473300 & 1.02129100 & 2.93173100\end{array}$




\begin{tabular}{|c|c|c|c|}
\hline $\mathrm{C}$ & 1.09880600 & -2.91076600 & -3.98680000 \\
\hline $\mathrm{H}$ & 5.20322100 & -1.24097900 & -2.11569400 \\
\hline $\mathrm{H}$ & -5.34950900 & 3.89541600 & -0.49951500 \\
\hline $\mathrm{H}$ & 3.77469500 & 2.78012700 & -2.69959700 \\
\hline $\mathrm{H}$ & -1.54832700 & 5.43652900 & -1.81148900 \\
\hline $\mathrm{H}$ & 5.99498900 & 3.44816900 & -3.57118700 \\
\hline $\mathrm{H}$ & -2.59547600 & 7.62870300 & -2.29023300 \\
\hline $\mathrm{H}$ & 7.42847300 & -0.58126000 & -2.98135800 \\
\hline $\mathrm{H}$ & -6.40309500 & 6.08655100 & -0.96021500 \\
\hline $\mathrm{H}$ & 4.68450800 & -3.23864400 & 1.35342000 \\
\hline $\mathrm{H}$ & -5.79701700 & 1.99845000 & 2.85061100 \\
\hline $\mathrm{H}$ & 2.78030800 & -1.51223800 & 4.80882300 \\
\hline $\mathrm{H}$ & -2.54396700 & 1.18929100 & 5.55453800 \\
\hline $\mathrm{H}$ & 4.68107200 & -2.23478400 & 6.22491700 \\
\hline $\mathrm{H}$ & -3.94247700 & 1.91986300 & 7.46419700 \\
\hline $\mathrm{H}$ & 6.59632900 & -3.95464100 & 2.75820200 \\
\hline $\mathrm{H}$ & -7.19669400 & 2.75000200 & 4.75005900 \\
\hline $\mathrm{H}$ & -5.05046300 & 7.98072500 & 51200 \\
\hline $\mathrm{H}$ & 7.84749400 & 1.76634700 & -3.75731500 \\
\hline $\mathrm{H}$ & -6.29853400 & 2.70195600 & 7.09236900 \\
\hline $\mathrm{H}$ & 6.60641000 & -3.49238500 & 5.22261900 \\
\hline $\mathrm{H}$ & -2.53288600 & 1.19900300 & -2.71634800 \\
\hline $\mathrm{H}$ & -4.00758900 & -4.74739000 & -1.09361500 \\
\hline $\mathrm{H}$ & -2.98180100 & -4.25503100 & -2.21285700 \\
\hline $\mathrm{H}$ & -1.40807300 & -2.86101600 & 3.17289200 \\
\hline $\mathrm{H}$ & -1.73307200 & -4.83365000 & 2.38661800 \\
\hline $\mathrm{H}$ & -0.08813200 & -2.35267100 & 3.90202500 \\
\hline $\mathrm{H}$ & -0.64404000 & -4.05837000 & -2.14221200 \\
\hline $\mathrm{H}$ & -3.14369600 & -1.61413700 & 2.26857100 \\
\hline $\mathrm{H}$ & -3.45794400 & -3.25205900 & -4.06491900 \\
\hline $\mathrm{H}$ & -0.55285400 & -0.52759100 & -5.12393100 \\
\hline $\mathrm{H}$ & 0.74683600 & 2.09281500 & -3.57042900 \\
\hline $\mathrm{H}$ & -0.77138100 & 0.71298700 & -4.15004000 \\
\hline $\mathrm{O}$ & -2.72272600 & -1.70975100 & -1.02173500 \\
\hline $\mathrm{O}$ & -5.10955500 & -1.21500900 & 0.58090500 \\
\hline $\mathrm{O}$ & -3.86417700 & 0.92710600 & 1.82034500 \\
\hline $\mathrm{O}$ & -4.83612500 & 0.10064200 & -1.90665800 \\
\hline $\mathrm{O}$ & -3.83607600 & -0.92701700 & -3.64138900 \\
\hline $\mathrm{O}$ & -1.21849000 & -0.10931300 & -4.56074600 \\
\hline $\mathrm{O}$ & -0.04626200 & -2.45983000 & -4.23520000 \\
\hline $\mathrm{O}$ & 2.06197800 & 1.17717900 & -1.89588800 \\
\hline $\mathrm{O}$ & 1.65200100 & -2.99392300 & -2.86093800 \\
\hline $\mathrm{O}$ & 2.83811300 & -0.89367900 & -1.53250000 \\
\hline $\mathrm{O}$ & 1.36920800 & -4.30743000 & -0.40849100 \\
\hline
\end{tabular}




$\begin{array}{lrrr}\mathrm{O} & 2.51153500 & -2.24367700 & 0.90883800 \\ \mathrm{O} & 1.46336900 & -1.34966900 & 2.68094000 \\ \mathrm{O} & -0.61138600 & -5.08883600 & 0.32851000 \\ \mathrm{O} & -0.32487200 & -2.21838000 & 0.64905700 \\ \mathrm{O} & -0.79690700 & -3.23927300 & -1.65467700 \\ \mathrm{O} & -2.68807900 & -3.37616700 & -3.49722400 \\ \mathrm{O} & -3.06226200 & -4.67642300 & -1.28288800 \\ \mathrm{O} & -0.03324200 & -0.80099000 & -1.90237900 \\ \mathrm{O} & -2.15345900 & 0.54885900 & -2.11189400 \\ \mathrm{O} & -1.54858400 & 3.01539800 & -1.09129500 \\ \mathrm{O} & -0.13057300 & 1.85112000 & -3.24990600 \\ \mathrm{O} & -3.58277400 & 2.23946100 & -0.55546700 \\ \mathrm{O} & -2.11232800 & -3.95525600 & 2.26115600 \\ \mathrm{O} & -0.94167700 & -2.04206400 & 3.56935700 \\ \mathrm{O} & -2.57037100 & -1.44817500 & 1.50972000 \\ \mathrm{O} & -4.37442500 & -3.34342200 & 0.64125000 \\ \mathrm{O} & -2.07051800 & 0.66586500 & 3.14219000 \\ \mathrm{O} & 1.02278600 & -0.03913100 & 0.32607800 \\ \mathrm{O} & -1.42420600 & 0.55609000 & 0.34992900 \\ \mathrm{Zr} & 1.14813400 & -2.11324300 & -0.84834500 \\ \mathrm{Zr} & -1.76134800 & -1.58365100 & -2.87983800 \\ \mathrm{Zr} & -2.07682500 & -3.29789600 & 0.25952600 \\ \mathrm{Zr} & -0.53283300 & -0.55516300 & 1.85169800 \\ \mathrm{Zr} & -3.38331100 & 0.06589800 & -0.16958300 \\ \mathrm{H} & -0.11865800 & 6.45343700 & 0.37262600 \\ \mathrm{H} r & -0.65239200 & 4.76430100 & 0.58300300 \\ \mathrm{H} & 1.08083600 & -6.20003800 & 0.17742600 \\ \mathrm{H} & 1.71512800 & -3.24375200 & -4.85837400 \\ \mathrm{H} & -6.30203400 & -2.81115100 & 0.90045300 \\ \mathrm{H} & -5.65317400 & -0.05225900 & -3.74520700 \\ \mathrm{H} & 1.84448000 & 0.43697900 & 0.59829700 \\ \mathrm{O} & 0.36881000 & 1.43344500 & 2.60829700 \\ \mathrm{H} & -0.37438800 & 1.89284200 & 3.02991000 \\ \mathrm{O} & 0.81536900 & 2.65234800 & 0.19169500 \\ \mathrm{P} & 1.99652100 & 3.59859700 & 0.48671500 \\ \mathrm{O} & 3.21337900 & 3.73543600 & -0.54034600 \\ \mathrm{O} & 1.36146200 & 5.07606800 & 0.15580900 \\ \mathrm{O} & 2.97979500 & 1.81376800 & 0.99421600 \\ \mathrm{H} & 3.72281500 & 4.98339600 & -0.99787700 \\ \mathrm{H} & 4.70852100 & 4.76386000 & -1.42680300 \\ \mathrm{H} & 3.06733700 & 5.42406400 & -1.75983500 \\ \mathrm{H} & & & \\ \mathrm{H} & & & \end{array}$




\begin{tabular}{|c|c|c|c|}
\hline $\mathrm{H}$ & 0.29537900 & 5.58533000 & \\
\hline $\mathrm{C}$ & 4.28790100 & 1.64263600 & 1.04601600 \\
\hline $\mathrm{C}$ & 5.19964300 & 2.70546600 & 1.27650800 \\
\hline $\mathrm{C}$ & 4.82461000 & 0.34396600 & 0.87459900 \\
\hline $\mathrm{C}$ & 6.56676900 & 2.48953300 & 1.25919800 \\
\hline $\mathrm{H}$ & 4.81949100 & 3.71213900 & 1.45868300 \\
\hline $\mathrm{C}$ & 6.18908800 & 0.12616000 & 0.86200200 \\
\hline $\mathrm{H}$ & 4.14026800 & -0.48660000 & 0.69200600 \\
\hline $\mathrm{C}$ & 7.06154200 & 1.20300700 & 1.03228300 \\
\hline $\mathrm{H}$ & 7.27420100 & 3.30487600 & 1.41387300 \\
\hline $\mathrm{H}$ & 6.60361000 & -0.86832300 & 0.69199400 \\
\hline $\mathrm{N}$ & 8.49438600 & 0.98604100 & 0.94780300 \\
\hline $\mathrm{O}$ & 9.23213500 & 1.94866000 & 1.12296900 \\
\hline $\mathrm{O}$ & 8.88821900 & -0.14744100 & 0.69666200 \\
\hline $\mathrm{O}$ & 2.24934000 & 3.91851900 & 2.05629000 \\
\hline $\mathrm{H}$ & 2.61461600 & 3.11702200 & 2.46668700 \\
\hline $\mathrm{H}$ & 0.56252600 & 1.93736400 & 1.77903900 \\
\hline \multicolumn{4}{|c|}{ NU-1000 DMPA Binding } \\
\hline $\mathrm{C}$ & 5.57492000 & 3.30090900 & -0.61556300 \\
\hline $\mathrm{C}$ & -5.76740500 & 2.59181000 & -0.94240900 \\
\hline $\mathrm{C}$ & 4.20818800 & 3.61602700 & -0.64596900 \\
\hline $\mathrm{C}$ & -4.45999900 & 3.09063500 & -0.84119400 \\
\hline $\mathrm{C}$ & 3.82025300 & 4.96340000 & -0.68960700 \\
\hline $\mathrm{C}$ & -4.25947900 & 4.47863600 & -0.87802400 \\
\hline $\mathrm{C}$ & 4.77907200 & 5.97097900 & -0.73382700 \\
\hline $\mathrm{C}$ & -5.33621400 & 5.34502200 & -1.04590300 \\
\hline $\mathrm{C}$ & 6.15387000 & 5.66442900 & -0.73854900 \\
\hline $\mathrm{C}$ & -6.64979400 & 4.85262500 & -1.17582500 \\
\hline $\mathrm{C}$ & 6.53096900 & 4.31083800 & -0.65710600 \\
\hline $\mathrm{C}$ & -6.84321600 & 3.45973500 & -1.10195400 \\
\hline $\mathrm{C}$ & 5.66500700 & -0.86953800 & 3.20313400 \\
\hline $\mathrm{C}$ & -5.66020800 & -1.45688100 & 2.80669000 \\
\hline $\mathrm{C}$ & 4.31486800 & -0.94279500 & 3.57667700 \\
\hline $\mathrm{C}$ & -4.34344700 & -1.32794300 & 3.27522200 \\
\hline $\mathrm{C}$ & 3.98962000 & -1.11173700 & 4.93024000 \\
\hline $\mathrm{C}$ & -4.08858900 & -1.51432700 & 4.64247600 \\
\hline $\mathrm{C}$ & 4.99340500 & -1.23647300 & 5.88587100 \\
\hline $\mathrm{C}$ & -5.12319600 & -1.84164400 & 5.51590600 \\
\hline $\mathrm{C}$ & 6.35225800 & -1.19088100 & 5.51808000 \\
\hline $\mathrm{C}$ & -6.44659800 & -1.98228900 & 5.05387400 \\
\hline $\mathrm{C}$ & 6.66679600 & -0.98628600 & 4.16134300 \\
\hline $\mathrm{C}$ & -6.69390800 & -1.77371800 & 3.68325300 \\
\hline $\mathrm{C}$ & -3.31606100 & 2.13852700 & -0.72970700 \\
\hline
\end{tabular}




$\begin{array}{lrrr}\mathrm{C} & -3.00116400 & -0.98128200 & -4.03289700 \\ \mathrm{C} & -2.90285600 & -4.19759000 & -0.99453000 \\ \mathrm{C} & 3.58227700 & -3.75567100 & -0.59618500 \\ \mathrm{C} & 3.25399800 & -0.84730500 & 2.53284700 \\ \mathrm{C} & 3.18018600 & 2.53952600 & -0.65295700 \\ \mathrm{C} & -3.23315000 & -1.06976800 & 2.31462500 \\ \mathrm{C} & 3.45615100 & -0.36907800 & -3.78603800 \\ \mathrm{H} & 5.88336800 & 2.25486700 & -0.56749500 \\ \mathrm{H} & -5.93583500 & 1.51442100 & -0.90345100 \\ \mathrm{H} & 2.75852000 & 5.21606200 & -0.70006800 \\ \mathrm{H} & -3.24615600 & 4.87560500 & -0.78783300 \\ \mathrm{H} & 4.45927200 & 7.01523100 & -0.77816400 \\ \mathrm{H} & -5.15678900 & 6.42248900 & -1.08444000 \\ \mathrm{H} & 7.59162500 & 4.04732600 & -0.64033000 \\ \mathrm{H} & -7.85407900 & 3.05207900 & -1.18505600 \\ \mathrm{H} & 5.92353500 & -0.72748900 & 2.15250900 \\ \mathrm{H} & -5.86710300 & -1.31517000 & 1.74433600 \\ \mathrm{H} & 2.94006400 & -1.15652700 & 5.22839700 \\ \mathrm{H} & -3.06741300 & -1.41558300 & 5.01541500 \\ \mathrm{H} & 4.72276100 & -1.37624100 & 6.93544700 \\ \mathrm{H} & -4.90290000 & -1.99248000 & 6.57571700 \\ \mathrm{H} & 7.71411800 & -0.93337400 & 3.85325800 \\ \mathrm{O} & -3.42496800 & -1.03234700 & -2.85073000 \\ \mathrm{H} & -7.71246800 & -1.87167800 & 3.29892900 \\ \mathrm{H} & -7.49329300 & 5.53107800 & -1.31426200 \\ \mathrm{H} & 6.90488100 & 6.45495300 & -0.78407300 \\ \mathrm{H} & -7.25614200 & -2.24131900 & 5.73830300 \\ \mathrm{H} & 7.13928800 & -1.29500400 & 6.26695200 \\ \mathrm{H} & -1.87666200 & 1.12137100 & -2.76629600 \\ \mathrm{H} & -0.47284200 & -5.00230500 & -2.75560300 \\ \mathrm{H} & 0.34296600 & -3.83488700 & -3.46037800 \\ \mathrm{H} & 0.25383500 & -3.57706200 & 2.30781200 \\ \mathrm{H} & 1.06235000 & -5.16675800 & 1.04608300 \\ \mathrm{H} & 1.02039100 & -2.75765200 & 3.42544200 \\ \mathrm{H} & 2.25266300 & -2.66793800 & -2.74983800 \\ \mathrm{H} & -1.72288800 & -3.03122400 & 1.20575300 \\ \mathrm{H} & -0.28506400 & -2.59714600 & -5.14379000 \\ \mathrm{H} & 1.04972300 & 1.21233800 & -4.82054800 \\ \mathrm{H} & -3.367921500 & 3.57094900 & -2.50228300 \\ \mathrm{H} & & -0.94920800 & -4.41538300 \\ \mathrm{H} & & & \\ \mathrm{H} & -536037000 & -1.92735200 & -1.79396000 \\ \mathrm{H} & -1.04035300 & -0.84914600 \\ \mathrm{H} & & & \end{array}$




$\begin{array}{lrrr}\mathrm{O} & 0.17750300 & 1.14322600 & -4.41174200 \\ \mathrm{O} & 2.29297900 & -0.41750700 & -4.24678600 \\ \mathrm{O} & 1.97830100 & 2.87766600 & -0.76869700 \\ \mathrm{O} & 3.81311300 & -0.53947000 & -2.59286900 \\ \mathrm{O} & 3.59916700 & 1.34423700 & -0.56089400 \\ \mathrm{O} & 3.86524200 & -2.53488800 & -0.62018800 \\ \mathrm{O} & 3.63405600 & -0.65199700 & 1.34603600 \\ \mathrm{O} & 2.04903300 & -0.97148900 & 2.89917400 \\ \mathrm{O} & 2.44522700 & -4.28558100 & -0.62869600 \\ \mathrm{O} & 1.22630300 & -1.81276500 & 0.34262400 \\ \mathrm{O} & 1.65348200 & -2.19094900 & -2.16720200 \\ \mathrm{O} & 0.37505500 & -2.54651200 & -4.44590600 \\ \mathrm{O} & 0.35553700 & -4.50916700 & -2.70254700 \\ \mathrm{O} & 1.15388700 & 0.28564900 & -1.69769900 \\ \mathrm{O} & -1.31223200 & 0.56669800 & -2.21859000 \\ \mathrm{O} & -2.16200800 & 2.61688200 & -0.64522500 \\ \mathrm{O} & -0.05917800 & 2.89957700 & -2.49945700 \\ \mathrm{O} & -3.59812100 & 0.89912600 & -0.77119100 \\ \mathrm{O} & 0.31061200 & -4.56770500 & 1.00329900 \\ \mathrm{O} & 0.17120000 & -2.81580300 & 2.96743100 \\ \mathrm{O} & -1.20670400 & -2.40699800 & 0.68618300 \\ \mathrm{O} & -1.69649300 & -4.54314900 & -1.00263200 \\ \mathrm{O} & -2.08314600 & 3.76669100 & 2.31451800 \\ \mathrm{O} & 0.13094500 & 3.12739000 & 3.45288600 \\ \mathrm{O} & -0.56133100 & 6.02827100 & 1.04916900 \\ \mathrm{O} & -2.06206700 & -0.92422300 & 2.77721100 \\ \mathrm{O} & 1.32138900 & 0.73866000 & 0.86679900 \\ \mathrm{O} & -1.04922000 & 0.14883800 & 0.31069500 \\ \mathrm{Zr} & 2.64718800 & -0.63576600 & -0.65730100 \\ \mathrm{Zr} & 0.18382600 & -0.83610100 & -3.24282400 \\ \mathrm{Zr} & 0.31738600 & -3.36767400 & -0.71048600 \\ \mathrm{Zr} & 0.03129200 & -0.85037900 & 1.75225200 \\ \mathrm{Zr} & -2.40241800 & -0.96949000 & -0.81847800 \\ \mathrm{Zr} & -0.03482700 & 1.77577000 & -0.72449300 \\ \mathrm{H} & 4.46276300 & -4.44031400 & -0.53456300 \\ \mathrm{H} & 4.27480600 & -0.11495500 & -4.50407500 \\ \mathrm{H} & -3.64641300 & -5.00626300 & -1.19416400 \\ \mathrm{O} & -3.78076700 & -0.96149700 & -4.83236700 \\ \mathrm{O} & -0.12567100 & 0.58981100 & 3.48359700 \\ \mathrm{O} & -1.02808100 & 0.50412700 & 3.81930900 \\ \mathrm{O} & -0.48414300 & 3.10452700 & 0.92344500 \\ \mathrm{O} & & & \\ \mathrm{O} & & & \end{array}$




$\begin{array}{llll}\mathrm{H} & -0.02908400 & 6.97555000 & 1.08281300 \\ \mathrm{H} & -0.31623000 & 5.50475700 & 0.12140900 \\ \mathrm{H} & -1.63562100 & 6.21536800 & 1.08287900 \\ \mathrm{C} & -2.75716800 & 2.52824900 & 2.48253700 \\ \mathrm{H} & -3.79264900 & 2.66877200 & 2.17652100 \\ \mathrm{H} & -2.31581100 & 1.73669900 & 1.86636800 \\ \mathrm{H} & -2.74177100 & 2.22455000 & 3.53344200 \\ \mathrm{H} & 0.08561100 & 1.58459200 & 3.50031900\end{array}$

Al $@ N U-1000$ Initial Node

$\begin{array}{lrrr}\mathrm{C} & 2.62741800 & 3.02558700 & 2.27443100 \\ \mathrm{C} & 3.98384200 & -1.40533300 & 2.44588300 \\ \mathrm{C} & 3.99048100 & -1.66846400 & -2.12374400 \\ \mathrm{C} & -2.48677000 & -3.37355800 & -2.34059100 \\ \mathrm{C} & -3.69861300 & 0.91071300 & -2.57732200 \\ \mathrm{C} & -3.86732500 & 1.08489500 & 2.20131600 \\ \mathrm{C} & 2.67172200 & 2.85245300 & -2.43418800 \\ \mathrm{C} & -2.89840900 & -3.18096000 & 2.46448600 \\ \mathrm{H} & 1.90804600 & 0.45969800 & 2.86034100 \\ \mathrm{H} & 2.27159600 & -4.15120700 & -1.37756300 \\ \mathrm{H} & 1.32020600 & -4.14974900 & -0.10049400 \\ \mathrm{H} & -0.01234600 & -0.22006200 & -4.14109200 \\ \mathrm{H} & -0.07758700 & -2.37975300 & -4.29443700 \\ \mathrm{H} & -1.14822800 & 0.84515600 & -4.51055900 \\ \mathrm{H} & -0.94254700 & -3.57167300 & 0.13778700 \\ \mathrm{H} & 1.94700600 & 0.26433400 & -2.86052500 \\ \mathrm{H} & 1.85439700 & -4.23960900 & 2.07917300 \\ \mathrm{H} & -0.61595600 & -2.10004900 & 4.04210000 \\ \mathrm{H} & -1.08494100 & 1.32804500 & 4.54213300 \\ \mathrm{H} & -0.03462300 & -0.62805800 & 4.10841300 \\ \mathrm{O} & 1.59628400 & -1.26293100 & 0.01210300 \\ \mathrm{O} & 4.17401900 & -0.68161600 & -1.36540100 \\ \mathrm{O} & 3.19384700 & 2.17420400 & -1.49673700 \\ \mathrm{O} & 4.09299200 & -0.46464900 & 1.62389900 \\ \mathrm{O} & 2.98685400 & -2.14054000 & 2.66820800 \\ \mathrm{O} & 0.21590700 & -1.58964000 & 3.93766700 \\ \mathrm{O} & -1.78890100 & -3.10912700 & 3.02402600 \\ \mathrm{O} & -2.80584500 & 1.64431500 & 2.58189600 \\ \mathrm{O} & -3.29818400 & -2.56199100 & 1.43309800 \\ \mathrm{O} & -3.93650700 & 0.11724400 & 1.38686500 \\ \mathrm{O} & -3.07832400 & -2.57803600 & -1.57762700 \\ \mathrm{O} & -3.95335900 & 0.13180500 & -1.62886700 \\ \mathrm{O} & -2.56346500 & 1.41959000 & -2.84174300 \\ & -1.25431100 & -3.44636400 & -2.58962200\end{array}$




$\begin{array}{lrrr}\mathrm{O} & -0.95550600 & -0.63401300 & -1.47046600 \\ \mathrm{O} & -0.67962500 & -2.64337800 & 0.06244500 \\ \mathrm{O} & 1.12413700 & -3.91433100 & 1.53584200 \\ \mathrm{O} & 1.34929700 & -4.04222600 & -1.10397800 \\ \mathrm{O} & -0.92516400 & 3.36720000 & 1.21831900 \\ \mathrm{O} & -0.98022600 & -0.58055800 & 1.45475800 \\ \mathrm{O} & 1.43736700 & 0.20230100 & 2.05511100 \\ \mathrm{O} & 1.42819500 & 2.82718900 & 2.61427700 \\ \mathrm{O} & -0.43152200 & 0.96806400 & 3.93154100 \\ \mathrm{O} & 3.26456000 & 2.41836700 & 1.36726300 \\ \mathrm{O} & 0.46831900 & -1.72374000 & -3.84083700 \\ \mathrm{O} & -0.29308200 & 0.75382900 & -4.06760200 \\ \mathrm{O} & 1.43612700 & 0.11332900 & -2.05285600 \\ \mathrm{O} & 2.91627800 & -2.24473600 & -2.41956500 \\ \mathrm{O} & 1.46620000 & 2.80353100 & -2.79526000 \\ \mathrm{O} & -0.89302100 & 3.33646600 & -1.30372000 \\ \mathrm{O} & -1.86931800 & 1.25753100 & -0.07378300 \\ \mathrm{O} & 0.80149100 & 1.69731400 & -0.03303100 \\ \mathrm{Zr} & -2.48026000 & -0.92894500 & 0.00482900 \\ \mathrm{Zr} & 0.66504000 & -1.91228000 & 1.74949500 \\ \mathrm{Zr} & 0.56768100 & -2.00757100 & -1.78997100 \\ \mathrm{Zr} & -0.46727400 & 1.35330400 & -1.88077400 \\ \mathrm{Zr} & 2.64595200 & 0.54955700 & 0.00863600 \\ \mathrm{Zr} & -0.51863500 & 1.40315700 & 1.92397700 \\ \mathrm{O} & 1.65912000 & 4.66634000 & -0.07066400 \\ \mathrm{Cl} & 2.57490400 & 4.37961600 & -0.03125000 \\ \mathrm{H} & -0.41998700 & -1.92517500 & 3.98733600 \\ \mathrm{O} & -3.80022800 & 3.56212200 & -0.09811100 \\ \mathrm{H} & -4.11918400 & 4.46125900 & -0.03648200 \\ \mathrm{Al} & -2.19685900 & 3.01654900 & -0.04613100 \\ \mathrm{Al} & 0.40270900 & 3.52217400 & -0.02135500 \\ \mathrm{H} & -3.12920600 & -4.10940400 & -2.88257400 \\ \mathrm{H} & -3.65819500 & -3.87530800 & 2.90771700 \\ \mathrm{H} & 4.91188000 & -2.08252000 & -2.60144400 \\ \mathrm{H} & 4.88912300 & -1.62230000 & 3.06304000 \\ \mathrm{H} & 3.33297500 & 3.50425600 & -2.96598400 \\ \mathrm{H} & 3.15636800 & 3.77626700 & 2.82360700 \\ \mathrm{H} & -4.50292600 & 1.16691600 & -3.23487000 \\ \mathrm{H} & -4.78635700 & 1.43692100 & 2.62134800 \\ \mathrm{C} & 2.91529600 & 1.84014900 & -3.95624400\end{array}$




\begin{tabular}{|c|c|c|c|}
\hline $\mathrm{C}$ & -1.46313700 & 1.06454100 & -3.62875500 \\
\hline $\mathrm{C}$ & -0.53379400 & -3.51532300 & -2.60145200 \\
\hline $\mathrm{C}$ & -1.36996900 & 2.55124500 & 2.86378900 \\
\hline $\mathrm{C}$ & 3.76308600 & -2.84015300 & -3.13700300 \\
\hline $\mathrm{H}$ & 1.88173400 & -2.31139500 & 2.56954900 \\
\hline $\mathrm{H}$ & 5.26105900 & 2.48341000 & 0.33302600 \\
\hline $\mathrm{H}$ & 5.29428100 & 0.99681100 & -0.23520000 \\
\hline $\mathrm{H}$ & 0.29651200 & 3.83666800 & -1.01394400 \\
\hline $\mathrm{H}$ & 2.23655300 & 4.24258300 & -1.90442200 \\
\hline $\mathrm{H}$ & -1.23342700 & 3.92129300 & -1.54409900 \\
\hline $\mathrm{H}$ & 4.16863900 & 0.00818900 & -2.03958200 \\
\hline $\mathrm{H}$ & 0.70242000 & 3.11110700 & 1.18838900 \\
\hline $\mathrm{H}$ & 6.05382500 & -0.89039700 & 0.73369100 \\
\hline $\mathrm{H}$ & 3.80677600 & -3.79521800 & -0.32049200 \\
\hline $\mathrm{H}$ & 0.63952000 & -4.89185000 & 0.46169400 \\
\hline $\mathrm{H}$ & 2.62384200 & -3.89372700 & 0.72706500 \\
\hline $\mathrm{O}$ & 2.72665200 & 0.53542200 & 1.03250900 \\
\hline $\mathrm{O}$ & 2.59670100 & 2.51862700 & 3.25502000 \\
\hline $\mathrm{O}$ & -0.36577500 & 1.92508500 & 3.32299000 \\
\hline $\mathrm{O}$ & 3.08525600 & -0.35504600 & 3.95733500 \\
\hline $\mathrm{O}$ & 4.56422000 & -1.42221100 & 2.61690200 \\
\hline $\mathrm{O}$ & 3.55620000 & -3.52786300 & 0.59012800 \\
\hline $\mathrm{O}$ & 4.15358200 & -3.04553600 & -1.97316700 \\
\hline $\mathrm{O}$ & -0.65164600 & -3.63902400 & -1.35424900 \\
\hline $\mathrm{O}$ & 2.82723200 & -2.07982100 & -3.52770400 \\
\hline $\mathrm{O}$ & 0.14820000 & -2.62506600 & -3.19039900 \\
\hline $\mathrm{O}$ & 2.18796800 & 0.82583900 & -4.03923300 \\
\hline $\mathrm{O}$ & -0.58966600 & 0.20985800 & -3.90756500 \\
\hline $\mathrm{O}$ & -1.66679300 & 1.57872300 & -2.48348600 \\
\hline $\mathrm{O}$ & 3.28273800 & 2.45518300 & -2.92038000 \\
\hline $\mathrm{O}$ & 1.05828100 & 1.08414300 & -1.43228900 \\
\hline $\mathrm{O}$ & 3.36695700 & 0.01861000 & -1.49820800 \\
\hline $\mathrm{O}$ & 5.41545900 & -0.64630000 & 0.05040500 \\
\hline $\mathrm{O}$ & 4.96596400 & 1.93505800 & -0.40819800 \\
\hline $\mathrm{O}$ & -1.99072100 & -2.05355900 & 0.59827700 \\
\hline $\mathrm{O}$ & 1.71118800 & -1.70128900 & -0.74542400 \\
\hline $\mathrm{O}$ & 1.80108700 & -1.64923600 & 1.86900400 \\
\hline $\mathrm{O}$ & -0.50487200 & -2.57550300 & 2.90896200 \\
\hline $\mathrm{O}$ & 0.99619900 & -4.08859200 & 0.85733100 \\
\hline $\mathrm{O}$ & 0.11188700 & -0.78922600 & 4.14315800 \\
\hline $\mathrm{O}$ & 1.92719400 & 3.90804400 & -1.05200700 \\
\hline $\mathrm{O}$ & -0.65091900 & 3.51717900 & -0.87769700 \\
\hline $\mathrm{O}$ & 0.90922000 & 2.21783800 & 0.87933800 \\
\hline $\mathrm{O}$ & 3.42643400 & 3.36649300 & 1.32405700 \\
\hline
\end{tabular}




\begin{tabular}{|c|c|c|c|}
\hline $\mathrm{O}$ & -1.76233700 & 2.53739700 & 1.66716400 \\
\hline $\mathrm{O}$ & -2.56221900 & 0.35420600 & 0.01764600 \\
\hline $\mathrm{O}$ & -0.62009000 & -0.79751600 & -1.21840000 \\
\hline $\mathrm{O}$ & -0.26740900 & -0.09256100 & 1.33900300 \\
\hline $\mathrm{Zr}$ & 1.22420600 & -0.72709700 & -2.54212800 \\
\hline $\mathrm{Zr}$ & 3.47455400 & -1.28213900 & 0.36949000 \\
\hline $\mathrm{Zr}$ & 2.68608500 & 2.04330800 & -0.58553200 \\
\hline $\mathrm{Zr}$ & -0.74607500 & 1.29961500 & -0.41337300 \\
\hline $\mathrm{Zr}$ & 1.34668600 & 0.58536500 & 2.60229200 \\
\hline $\mathrm{Zr}$ & 0.09213500 & -2.27196400 & 0.45451300 \\
\hline $\mathrm{O}$ & -2.88259200 & -0.30109400 & 3.02294300 \\
\hline $\mathrm{H}$ & -2.38324500 & -0.00670500 & 3.78976400 \\
\hline $\mathrm{O}$ & -2.85640000 & -1.53194200 & -2.68418200 \\
\hline $\mathrm{H}$ & -2.10994500 & -1.42514900 & -3.28320900 \\
\hline $\mathrm{Al}$ & -2.47078700 & -1.21992500 & -1.01913800 \\
\hline $\mathrm{Al}$ & -2.05626700 & -0.50589900 & 1.53494700 \\
\hline $\mathrm{H}$ & 3.28028200 & 2.26749400 & -4.92171900 \\
\hline $\mathrm{H}$ & 4.29045300 & -3.38262900 & -3.96375800 \\
\hline $\mathrm{H}$ & 3.81485100 & 4.11230400 & 3.15028400 \\
\hline $\mathrm{H}$ & 4.73305500 & -1.30096200 & 4.61689300 \\
\hline $\mathrm{O}$ & -4.33377600 & -1.67651800 & -0.59668600 \\
\hline $\mathrm{P}$ & -5.67743700 & -1.70160000 & 0.03000900 \\
\hline $\mathrm{O}$ & -6.80913100 & -2.05434100 & -1.01504800 \\
\hline $\mathrm{O}$ & -5.90600800 & -2.72480800 & 1.21123500 \\
\hline $\mathrm{O}$ & -6.08098400 & -0.28016100 & 0.70345600 \\
\hline $\mathrm{C}$ & -8.18053900 & -2.17328000 & -0.63383700 \\
\hline $\mathrm{H}$ & -8.74904400 & -2.32275900 & -1.55598200 \\
\hline $\mathrm{H}$ & -8.32249300 & -3.03524200 & 0.03037400 \\
\hline $\mathrm{H}$ & -8.52955100 & -1.25919000 & -0.13397100 \\
\hline $\mathrm{C}$ & -4.94413700 & -2.80627200 & 2.28552000 \\
\hline $\mathrm{H}$ & -5.26881000 & -3.63197300 & 2.92386100 \\
\hline $\mathrm{H}$ & -3.94076500 & -3.01083600 & 1.88570000 \\
\hline $\mathrm{H}$ & -4.92884000 & -1.87003900 & 2.85840000 \\
\hline $\mathrm{C}$ & -5.54346400 & 0.92593500 & 0.29314300 \\
\hline $\mathrm{C}$ & -5.23979200 & 1.83924100 & 1.30121100 \\
\hline $\mathrm{C}$ & -5.29612400 & 1.19915500 & -1.05333500 \\
\hline $\mathrm{C}$ & -4.62225500 & 3.03107500 & 0.96483700 \\
\hline $\mathrm{H}$ & -5.44587900 & 1.57601500 & 2.33875900 \\
\hline $\mathrm{C}$ & -4.64927100 & 2.37958200 & -1.38766000 \\
\hline $\mathrm{H}$ & -5.54879400 & 0.47103400 & -1.82723100 \\
\hline $\mathrm{C}$ & -4.30743000 & 3.27257300 & -0.37261100 \\
\hline $\mathrm{H}$ & -4.33133200 & 3.75754500 & 1.72132100 \\
\hline $\mathrm{H}$ & -4.38236000 & 2.60631000 & -2.41897500 \\
\hline $\mathrm{N}$ & -3.55190700 & 4.47369700 & -0.70308700 \\
\hline
\end{tabular}




$\begin{array}{llrr}\mathrm{O} & -3.07622900 & 4.54894900 & -1.83804900 \\ \mathrm{O} & -3.40519600 & 5.32069200 & 0.15746600 \\ \mathrm{H} & -2.09187600 & 1.40509000 & -4.42475500 \\ \mathrm{H} & -1.90777300 & 3.15395400 & 3.56550800 \\ \mathrm{H} & -0.83010600 & -2.39549900 & 4.85653100 \\ \mathrm{H} & -1.02765200 & -4.23886600 & -3.21585400\end{array}$

$\mathrm{Al}_{2} @ \mathrm{NU}-1000$ Pre-Reaction Complex

$\begin{array}{lrrr}\mathrm{C} & 0.22655600 & 1.07362600 & 4.41521600 \\ \mathrm{C} & 4.68626400 & 1.15578600 & 3.14770200 \\ \mathrm{C} & 3.72171000 & 3.91361000 & -0.37610400 \\ \mathrm{C} & 2.37751600 & -1.24296600 & -4.43966900 \\ \mathrm{C} & -1.91061500 & -1.20247700 & -3.21913300 \\ \mathrm{C} & -0.88373700 & -4.22957000 & 0.34126100 \\ \mathrm{C} & -0.83611400 & 3.96265700 & 0.84766800 \\ \mathrm{C} & 3.29177000 & -4.46879100 & -0.96787200 \\ \mathrm{H} & 2.26449100 & -0.31618400 & 3.31285600 \\ \mathrm{H} & 5.32522300 & 1.67401400 & -1.78635100 \\ \mathrm{H} & 5.25993000 & 0.15949800 & -1.30241000 \\ \mathrm{H} & 0.17546300 & 2.49215400 & -3.06976900 \\ \mathrm{H} & 1.94585000 & 1.98153800 & -4.25719800 \\ \mathrm{H} & -1.24921700 & 3.20268800 & -2.69423300 \\ \mathrm{H} & 3.85845700 & -1.61002300 & -1.96021200 \\ \mathrm{H} & 0.99947500 & 3.16109000 & -0.96681500 \\ \mathrm{H} & 6.13991600 & -0.77732000 & 0.54006300 \\ \mathrm{H} & 3.70877000 & -3.47168800 & 1.81312600 \\ \mathrm{H} & 0.68508300 & -3.49427500 & 3.38769700 \\ \mathrm{H} & 2.68581100 & -2.76275100 & 2.79765000 \\ \mathrm{O} & 2.91583300 & 0.83786200 & 0.24755200 \\ \mathrm{O} & 3.14402100 & 3.77855500 & 0.73342100 \\ \mathrm{O} & 0.21909200 & 3.65734200 & 1.48381200 \\ \mathrm{O} & 3.70348500 & 1.92442800 & 3.02233400 \\ \mathrm{O} & 4.95552700 & 0.11435400 & 2.49428500 \\ \mathrm{O} & 3.60112400 & -2.67183800 & 2.37059400 \\ \mathrm{O} & 3.84933400 & -3.94835600 & 0.01572400 \\ \mathrm{O} & -0.81562200 & -3.54253900 & 1.39406700 \\ \mathrm{O} & 2.31565700 & -4.02344500 & -1.64283800 \\ \mathrm{O} & -0.28839600 & -3.96903700 & -0.74611100 \\ \mathrm{O} & 1.63601100 & -2.01112100 & -3.78802100 \\ \mathrm{O} & -1.09693400 & -2.12915000 & -2.99472700 \\ \mathrm{O} & -1.93615200 & -0.07291100 & -2.63542700 \\ \mathrm{O} & 2.90130000 & -0.16009300 & -4.06641000 \\ \mathrm{O} & 0.88735500 & -0.06403100 & -1.80854500 \\ & 3.15324200 & -1.18682200 & -1.45093700\end{array}$




\begin{tabular}{|c|c|c|c|}
\hline $\mathrm{O}$ & 5.41130200 & -0.95412800 & -0.06976900 \\
\hline $\mathrm{O}$ & 4.92117800 & 0.81778300 & -1.98855600 \\
\hline $\mathrm{O}$ & -1.87240700 & -0.96591600 & 2.04078900 \\
\hline $\mathrm{O}$ & 1.61545700 & -1.87469400 & 0.39487900 \\
\hline $\mathrm{O}$ & 2.09812000 & -0.22692900 & 2.36404700 \\
\hline $\mathrm{O}$ & -0.02452000 & -0.09159400 & 4.00018200 \\
\hline $\mathrm{O}$ & 1.11110900 & -2.65099400 & 3.19673500 \\
\hline $\mathrm{O}$ & 0.78687800 & 2.00142500 & 3.76499900 \\
\hline $\mathrm{O}$ & 1.80617400 & 2.28534000 & -3.35045400 \\
\hline $\mathrm{O}$ & -0.74133400 & 2.36928400 & -2.67876900 \\
\hline $\mathrm{O}$ & 1.12916100 & 2.24751600 & -0.68024600 \\
\hline $\mathrm{O}$ & 3.68501500 & 3.16097300 & -1.37890400 \\
\hline $\mathrm{O}$ & -1.40257600 & 3.25130700 & -0.02362300 \\
\hline $\mathrm{O}$ & -2.49989200 & 0.60255600 & 0.13381200 \\
\hline $\mathrm{O}$ & -0.74805600 & -1.25752900 & -0.28870500 \\
\hline $\mathrm{O}$ & -0.05337900 & 0.84587900 & 1.21096800 \\
\hline $\mathrm{Zr}$ & 0.89517900 & -2.18199600 & -1.54485100 \\
\hline $\mathrm{Zr}$ & 3.53663800 & -1.05397300 & 0.79828800 \\
\hline $\mathrm{Zr}$ & 2.64115100 & 1.03302300 & -1.93173800 \\
\hline $\mathrm{Zr}$ & -0.73198300 & 0.91895700 & -0.92426400 \\
\hline $\mathrm{Zr}$ & 1.76145300 & 1.97732700 & 1.58215600 \\
\hline $\mathrm{Zr}$ & 0.17205500 & -1.37441100 & 1.85179300 \\
\hline $\mathrm{O}$ & -1.92847600 & 2.24356100 & 2.94198100 \\
\hline $\mathrm{H}$ & -1.10027000 & 2.71273500 & 3.08167100 \\
\hline $\mathrm{O}$ & -3.19384100 & -2.44875200 & -0.78482800 \\
\hline $\mathrm{H}$ & -2.54650700 & -2.79056900 & -1.41333900 \\
\hline $\mathrm{Al}$ & -2.51352500 & -1.26398000 & 0.32224300 \\
\hline $\mathrm{Al}$ & -1.94901900 & 0.87506300 & 1.87008200 \\
\hline $\mathrm{H}$ & 2.59871800 & -1.54406300 & -5.49247500 \\
\hline $\mathrm{H}$ & 3.68464400 & -5.45616700 & -1.32375200 \\
\hline $\mathrm{H}$ & 4.34862200 & 4.83253500 & -0.48017500 \\
\hline $\mathrm{H}$ & 5.42177100 & 1.42891700 & 3.94263300 \\
\hline $\mathrm{O}$ & -4.37752800 & -1.29093600 & 1.10349300 \\
\hline $\mathrm{P}$ & -5.69788600 & -1.49136000 & 0.39613400 \\
\hline $\mathrm{O}$ & -6.15028300 & -2.93112900 & -0.03870200 \\
\hline $\mathrm{O}$ & -6.89102900 & -1.03842800 & 1.35516600 \\
\hline $\mathrm{O}$ & -5.90799100 & -0.58188000 & -0.90498700 \\
\hline $\mathrm{C}$ & -5.73472300 & -3.70741300 & -1.17151100 \\
\hline $\mathrm{H}$ & -6.58427800 & -4.35279300 & -1.41751600 \\
\hline $\mathrm{H}$ & -5.49183700 & -3.05821400 & -2.01929200 \\
\hline $\mathrm{H}$ & -4.85262200 & -4.29845600 & -0.91196600 \\
\hline $\mathrm{C}$ & -7.13919700 & -1.71667900 & 2.58381600 \\
\hline $\mathrm{H}$ & -7.47359600 & -2.74627600 & 2.39849600 \\
\hline $\mathrm{H}$ & -6.24220400 & -1.73520600 & 3.22000700 \\
\hline
\end{tabular}




$\begin{array}{lrrr}\mathrm{H} & -7.93275300 & -1.16527900 & 3.09656700 \\ \mathrm{C} & -5.34235400 & 0.67876100 & -1.05735300 \\ \mathrm{C} & -5.40385500 & 1.62430400 & -0.03372400 \\ \mathrm{C} & -4.71203800 & 0.94295000 & -2.26640800 \\ \mathrm{C} & -4.77428300 & 2.84632400 & -0.20899800 \\ \mathrm{H} & -5.90494200 & 1.38745800 & 0.90503000 \\ \mathrm{C} & -4.07472700 & 2.16328600 & -2.44204300 \\ \mathrm{H} & -4.68600800 & 0.17662300 & -3.03978500 \\ \mathrm{C} & -4.10567600 & 3.09517600 & -1.40731500 \\ \mathrm{H} & -4.76377800 & 3.59917000 & 0.57917800 \\ \mathrm{H} & -3.53963800 & 2.38251700 & -3.36535000 \\ \mathrm{~N} & -3.40124800 & 4.36398000 & -1.56857800 \\ \mathrm{O} & -2.60971100 & 4.46444900 & -2.50703200 \\ \mathrm{O} & -3.63322700 & 5.25404600 & -0.77719000 \\ \mathrm{H} & -2.64759200 & -1.36598600 & -3.97744900 \\ \mathrm{H} & -1.25734800 & 4.92068600 & 1.07049100 \\ \mathrm{H} & -0.05404500 & 1.28623000 & 5.42564100 \\ \mathrm{H} & -1.47075400 & -5.12352100 & 0.37505100 \\ \mathrm{O} & -3.85667600 & 0.79238400 & 2.60955800 \\ \mathrm{H} & -4.12179600 & -0.14088100 & 2.64978600 \\ \mathrm{H} & -3.73835600 & 1.14735300 & 3.50246200\end{array}$

$\mathrm{Al}_{2} @ \mathrm{NU}-1000 \mathrm{TS} 1$

$\begin{array}{lrrr}\text { C } & 1.30545200 & 1.38574000 & -3.63291400 \\ \mathrm{C} & -3.25077400 & 2.22062400 & -3.84765900 \\ \mathrm{C} & -3.12296800 & 4.41911900 & 0.16525100 \\ \mathrm{C} & -4.04479900 & -1.23620700 & 3.64049600 \\ \mathrm{C} & 0.35516400 & -1.92205800 & 3.86287700 \\ \mathrm{C} & 0.14036900 & -4.36833000 & -0.24368200 \\ \mathrm{C} & 1.52975300 & 3.68956000 & 0.47138600 \\ \mathrm{C} & -4.21561200 & -3.90627700 & -0.37655800 \\ \mathrm{H} & -1.19083600 & 0.30398200 & -3.45353400 \\ \mathrm{H} & -5.46743500 & 2.42554200 & 0.65396800 \\ \mathrm{H} & -5.46196500 & 0.98047600 & -0.01637200 \\ \mathrm{H} & -1.07968000 & 1.98678200 & 3.63684700 \\ \mathrm{H} & -3.19561000 & 1.87051100 & 4.07491900 \\ \mathrm{H} & 0.13712200 & 1.25763900 & 4.37676600 \\ \mathrm{H} & -4.66689400 & -1.07645400 & 0.76955800 \\ \mathrm{H} & -0.91004400 & 3.08857800 & 1.53605100 \\ \mathrm{H} & -5.79468700 & 0.39044400 & -2.16305500 \\ \mathrm{H} & -3.55972500 & -2.60368000 & -2.96245700 \\ \mathrm{H} & -0.18542400 & -3.09579100 & -3.59644100 \\ \mathrm{H} & -2.17507100 & -2.04576000 & -3.48649900 \\ \mathrm{O} & -2.62974900 & 1.32223300 & -0.61569700\end{array}$




\begin{tabular}{|c|c|c|c|}
\hline $\mathrm{O}$ & -2.23205700 & 4.27226600 & -0.71092000 \\
\hline $\mathrm{O}$ & 0.71845200 & 3.65183700 & -0.50417700 \\
\hline $\mathrm{O}$ & -2.26243800 & 2.76989100 & -3.30550600 \\
\hline $\mathrm{O}$ & -3.88231500 & 1.19809300 & -3.47399700 \\
\hline $\mathrm{O}$ & -3.14199000 & -1.79804600 & -3.33808900 \\
\hline $\mathrm{O}$ & -4.32016000 & -3.20334900 & -1.39839100 \\
\hline $\mathrm{O}$ & 0.53923300 & -3.59162100 & -1.15056900 \\
\hline $\mathrm{O}$ & -3.46930100 & -3.71979000 & 0.63084300 \\
\hline $\mathrm{O}$ & -0.74009700 & -4.09351700 & 0.62454200 \\
\hline $\mathrm{O}$ & -3.25426100 & -2.07361500 & 3.15216500 \\
\hline $\mathrm{O}$ & -0.46702100 & -2.64985500 & 3.25830800 \\
\hline $\mathrm{O}$ & 0.75048900 & -0.77215200 & 3.49021200 \\
\hline $\mathrm{O}$ & -4.23787400 & -0.04378400 & 3.28390300 \\
\hline $\mathrm{O}$ & -1.62624700 & -0.16267500 & 1.82161000 \\
\hline $\mathrm{O}$ & -3.77061000 & -0.75839400 & 0.59190000 \\
\hline $\mathrm{O}$ & -5.34664500 & 0.03371100 & -1.38447300 \\
\hline $\mathrm{O}$ & -5.27351200 & 1.49402700 & 0.83199700 \\
\hline $\mathrm{O}$ & 2.07724100 & -1.22683700 & -1.07756300 \\
\hline $\mathrm{O}$ & -1.82408200 & -1.59836100 & -0.73829700 \\
\hline $\mathrm{O}$ & -1.33777100 & 0.27882300 & -2.49754500 \\
\hline $\mathrm{O}$ & 1.21854800 & 0.16214700 & -3.33577900 \\
\hline $\mathrm{O}$ & -0.54004200 & -2.23668500 & -3.34199700 \\
\hline $\mathrm{O}$ & 0.70821200 & 2.34403500 & -3.06500800 \\
\hline $\mathrm{O}$ & -2.65253500 & 2.17777400 & 3.33678900 \\
\hline $\mathrm{O}$ & -0.09340200 & 1.75379100 & 3.57994000 \\
\hline $\mathrm{O}$ & -1.08073400 & 2.25020500 & 1.08417400 \\
\hline $\mathrm{O}$ & -3.54421700 & 3.58874500 & 1.00575400 \\
\hline $\mathrm{O}$ & 1.65114300 & 2.80821000 & 1.36275700 \\
\hline $\mathrm{O}$ & 2.27584900 & 0.05165600 & 1.15905500 \\
\hline $\mathrm{O}$ & 0.25990200 & -1.47176900 & 0.74103500 \\
\hline $\mathrm{O}$ & 0.41853800 & 0.86937000 & -0.59724300 \\
\hline $\mathrm{Zr}$ & -1.83410300 & -2.21036700 & 1.27113200 \\
\hline $\mathrm{Zr}$ & -3.33146500 & -0.36420000 & -1.60518100 \\
\hline $\mathrm{Zr}$ & -3.11282800 & 1.25308100 & 1.54060500 \\
\hline $\mathrm{Zr}$ & 0.32214200 & 0.55034700 & 1.68465100 \\
\hline $\mathrm{Zr}$ & -0.96608000 & 2.35901400 & -1.34931400 \\
\hline $\mathrm{Zr}$ & 0.05779000 & -1.31930000 & -1.59409100 \\
\hline $\mathrm{O}$ & 3.56933500 & 1.17647200 & -1.44883900 \\
\hline $\mathrm{H}$ & 4.09851900 & 1.93696400 & -1.15957700 \\
\hline $\mathrm{O}$ & 2.15231500 & -3.28337700 & 1.66017400 \\
\hline $\mathrm{H}$ & 1.27881100 & -3.53409300 & 1.97721100 \\
\hline $\mathrm{Al}$ & 2.20877100 & -1.81598600 & 0.73223800 \\
\hline $\mathrm{Al}$ & 2.17262300 & 0.47523800 & -0.56961700 \\
\hline $\mathrm{H}$ & -4.65280600 & -1.57971800 & 4.51244900 \\
\hline
\end{tabular}




$\begin{array}{lrrr}\mathrm{H} & -4.85287900 & -4.82592900 & -0.31291200 \\ \mathrm{H} & -3.59856900 & 5.42953100 & 0.19905800 \\ \mathrm{H} & -3.62244900 & 2.69992600 & -4.78553600 \\ \mathrm{O} & 4.08387700 & -1.83514900 & 0.56356700 \\ \mathrm{P} & 5.12369200 & -2.01925900 & -0.55830300 \\ \mathrm{O} & 6.25944400 & -3.14860800 & -0.27415700 \\ \mathrm{O} & 4.37575100 & -3.06662000 & -1.62521800 \\ \mathrm{O} & 6.29077500 & -0.82589600 & -0.03188400 \\ \mathrm{C} & 7.22052900 & -3.07965900 & 0.76310400 \\ \mathrm{H} & 7.56318700 & -4.10784600 & 0.93364500 \\ \mathrm{H} & 8.07559300 & -2.45509400 & 0.47574800 \\ \mathrm{H} & 6.78922000 & -2.68813500 & 1.69463200 \\ \mathrm{C} & 4.03991100 & -4.37721000 & -1.21353000 \\ \mathrm{H} & 3.27290200 & -4.74376000 & -1.90737700 \\ \mathrm{H} & 4.91193400 & -5.04556000 & -1.25210000 \\ \mathrm{H} & 3.62135300 & -4.38661500 & -0.19317300 \\ \mathrm{C} & 6.01368600 & 0.44794200 & 0.25762100 \\ \mathrm{C} & 6.56389000 & 1.46076000 & -0.55463200 \\ \mathrm{C} & 5.19851800 & 0.79975600 & 1.34911500 \\ \mathrm{C} & 6.25934400 & 2.79359500 & -0.31883700 \\ \mathrm{H} & 7.20974600 & 1.16605700 & -1.38390000 \\ \mathrm{C} & 4.88431500 & 2.13053900 & 1.58500000 \\ \mathrm{H} & 4.78419400 & 0.01233400 & 1.97546600 \\ \mathrm{C} & 5.39921200 & 3.11460100 & 0.73791100 \\ \mathrm{H} & 6.65875900 & 3.59516300 & -0.94110400 \\ \mathrm{H} & 4.21906400 & 2.41868200 & 2.39906900 \\ \mathrm{~N} & 5.03505500 & 4.51620800 & 0.95582200 \\ \mathrm{O} & 4.33549600 & 4.77700600 & 1.92350300 \\ \mathrm{O} & 5.43823600 & 5.33700300 & 0.14495600 \\ \mathrm{H} & 0.75748200 & -2.29133200 & 4.78302300 \\ \mathrm{H} & 2.14379600 & 4.56336300 & 0.53708500 \\ \mathrm{H} & 1.94062700 & 1.62755100 & -4.45934100 \\ \mathrm{H} & 0.55901700 & -5.35276600 & -0.22062200 \\ \mathrm{O} & 4.82954900 & -0.87158900 & -1.87547200 \\ \mathrm{H} & 4.20275800 & -1.35887400 & -2.44563000 \\ \mathrm{H} & 4.25434500 & 0.28019300 & -1.65888500\end{array}$

$\begin{array}{lrrr}\mathrm{Al}_{2} @ N U-1000 \mathrm{INT} 1 & & \\ \mathrm{C} & -0.76851700 & -1.40828800 & 4.01178300 \\ \mathrm{C} & 3.85164000 & -1.01420500 & 3.97718400 \\ \mathrm{C} & 3.55755900 & 3.29326700 & 2.45653500 \\ \mathrm{C} & 3.40145600 & 1.34363600 & -3.95316900 \\ \mathrm{C} & -1.04721400 & 1.06315100 & -3.85242200 \\ \mathrm{C} & -0.68731700 & -3.49358300 & -2.43791400\end{array}$




\begin{tabular}{|c|c|c|c|}
\hline $\mathrm{C}$ & -1.15489800 & 3.04047500 & 2.507 \\
\hline C & 3.68102200 & -3.32050000 & -2.74369900 \\
\hline $\mathrm{H}$ & 1.55986000 & -2.15381200 & 2.75118400 \\
\hline $\mathrm{H}$ & 5.58266500 & 2.09784100 & 0.40039000 \\
\hline $\mathrm{H}$ & 5.47254000 & 0.57242800 & -0.03921200 \\
\hline $\mathrm{H}$ & 0.80286000 & 3.92284600 & -1.35621700 \\
\hline $\mathrm{H}$ & 2.87580000 & 3.97636300 & -2.13411800 \\
\hline $\mathrm{H}$ & -0.79476000 & 4.21241000 & -1.69647900 \\
\hline $\mathrm{H}$ & 4.35513700 & -0.43007200 & -1.83368100 \\
\hline $\mathrm{H}$ & 1.05437300 & 3.24789400 & 0.90746400 \\
\hline $\mathrm{H}$ & 5.97347400 & -1.30912100 & 1.11593100 \\
\hline $\mathrm{H}$ & 3.45939600 & -4.04392500 & 0.12176800 \\
\hline $\mathrm{H}$ & 0.15595500 & -4.74410300 & 0.74345200 \\
\hline $\mathrm{H}$ & 2.22448900 & -3.93622100 & 1.10327100 \\
\hline $\mathrm{O}$ & 2.79312600 & 0.46857800 & 1.09030500 \\
\hline $\mathrm{O}$ & 2.75580700 & 2.62012900 & 3.15442200 \\
\hline $\mathrm{O}$ & -0.25315900 & 2.35004400 & 3.07469000 \\
\hline $\mathrm{O}$ & 2.88454700 & -0.22447700 & 4.09334000 \\
\hline $\mathrm{O}$ & 4.30970200 & -1.54452400 & 2.93172700 \\
\hline $\mathrm{O}$ & 3.19112800 & -3.67784200 & 0.99308300 \\
\hline $\mathrm{O}$ & 3.98064100 & -3.47328900 & -1.54533900 \\
\hline $\mathrm{O}$ & -0.88781700 & -3.50512100 & -1.19518700 \\
\hline $\mathrm{O}$ & 2.85804500 & -2.49887100 & -3.24789000 \\
\hline $\mathrm{O}$ & 0.12057100 & -2.72971800 & -3.04483800 \\
\hline $\mathrm{O}$ & 2.57258000 & 0.40845000 & -4.00893100 \\
\hline $\mathrm{O}$ & -0.25900500 & 0.10163600 & -4.01175400 \\
\hline $\mathrm{O}$ & -1.25684700 & 1.68491900 & -2.76294700 \\
\hline $\mathrm{O}$ & 3.77578800 & 1.99640400 & -2.94318800 \\
\hline $\mathrm{O}$ & 1.34248000 & 0.99402300 & -1.51932200 \\
\hline $\mathrm{O}$ & 3.52982200 & -0.30076100 & -1.34573600 \\
\hline $\mathrm{O}$ & 5.39926100 & -1.05249500 & 0.38210100 \\
\hline $\mathrm{O}$ & 5.25437400 & 1.52008000 & -0.30337400 \\
\hline $\mathrm{O}$ & -2.11768500 & -1.65461600 & 0.53978400 \\
\hline $\mathrm{O}$ & 1.66448900 & -1.78480500 & -0.60644000 \\
\hline $\mathrm{O}$ & 1.58877500 & -1.53704300 & 2.00643500 \\
\hline $\mathrm{O}$ & -0.86396400 & -2.12924700 & 2.98021700 \\
\hline $\mathrm{O}$ & 0.54638800 & -3.95175900 & 1.12996000 \\
\hline $\mathrm{O}$ & -0.12427300 & -0.32612400 & 4.11864300 \\
\hline $\mathrm{O}$ & 2.48138500 & 3.73523400 & -1.28519900 \\
\hline $\mathrm{O}$ & -0.15142400 & 3.66668600 & -1.19848500 \\
\hline $\mathrm{O}$ & 1.18801300 & 2.31254600 & 0.69719500 \\
\hline $\mathrm{O}$ & 3.78086400 & 3.22024000 & 1.22437600 \\
\hline $\mathrm{O}$ & -1.47869800 & 2.97355500 & 1.29245500 \\
\hline $\mathrm{O}$ & -2.37934200 & 0.78468900 & -0.26960600 \\
\hline
\end{tabular}




$\begin{array}{lrrr}\mathrm{O} & -0.54362400 & -0.68023400 & -1.27013100 \\ \mathrm{O} & -0.26675500 & 0.19616100 & 1.27449600 \\ \mathrm{Zr} & 1.36189300 & -0.90298400 & -2.49374800 \\ \mathrm{Zr} & 3.38954700 & -1.45833600 & 0.60576700 \\ \mathrm{Zr} & 3.01355500 & 1.85201100 & -0.63775900 \\ \mathrm{Zr} & -0.47379800 & 1.51108300 & -0.66637800 \\ \mathrm{Zr} & 1.36145000 & 0.79917400 & 2.56816000 \\ \mathrm{Zr} & -0.05944100 & -2.08132000 & 0.49867600 \\ \mathrm{O} & -3.33196500 & 0.26465500 & 2.51212600 \\ \mathrm{H} & -3.80526600 & 1.11264700 & 2.35555900 \\ \mathrm{O} & -2.71394600 & -1.35425600 & -2.85784700 \\ \mathrm{H} & -1.91824600 & -1.34659300 & -3.39902600 \\ \mathrm{Al} & -2.48971600 & -0.89150900 & -1.19769400 \\ \mathrm{Al} & -2.03770100 & -0.03902800 & 1.26164200 \\ \mathrm{H} & 3.86486400 & 1.65234100 & -4.92164000 \\ \mathrm{H} & 4.19090900 & -3.97931600 & -3.49329800 \\ \mathrm{H} & 4.14611100 & 4.06293100 & 3.01285800 \\ \mathrm{H} & 4.37848200 & -1.28399900 & 4.92435600 \\ \mathrm{O} & -4.31202400 & -1.03330100 & -0.82883500 \\ \mathrm{P} & -5.24866200 & -1.60174800 & 0.24997800 \\ \mathrm{O} & -6.79188300 & -1.86545400 & -0.12289100 \\ \mathrm{H} & -3.14596700 & 4.73844900 & -0.83742200 \\ \mathrm{O} & -4.87643400 & -3.22363000 & 0.12482300 \\ \mathrm{O} & -2.92404800 & 5.62454100 & -0.02741000 \\ \mathrm{O} & -5.73072500 & 0.18183000 & 0.79984600 \\ \mathrm{C} & -7.79306200 & -0.89993700 & -0.38802400 \\ \mathrm{H} & -8.63196100 & -1.44978300 & -0.83045900 \\ \mathrm{H} & -8.12613500 & -0.40311700 & 0.53104700 \\ \mathrm{H} & -7.45550800 & -0.13790000 & -1.10367600 \\ \mathrm{C} & -4.95131900 & -3.85013700 & -1.13819800 \\ \mathrm{H} & -4.54327600 & -4.86313100 & -1.02876000 \\ \mathrm{H} & -5.99360500 & -3.93101600 & -1.48403100 \\ \mathrm{H} & -4.36419500 & -3.30983600 & -1.89951600 \\ \mathrm{C} & -5.34539400 & 1.32956100 & 0.30257900 \\ \mathrm{C} & -5.10397800 & 2.38773900 & 1.22756000 \\ \mathrm{C} & -5.11902500 & 1.59857900 & -1.07277600 \\ \mathrm{H} & -4.45443400 & 3.54510400 & 0.84074100 \\ \mathrm{H} & -5.43646100 & 2.25037400 & 2.26270000 \\ \mathrm{H} & -4.44792400 & 2.74212500 & -1.45557200 \\ \mathrm{H} & -4.37216900 & 0.84736200 & -1.81932800 \\ \mathrm{H} & & & \\ \mathrm{H} & & & \\ \mathrm{H} & & & \end{array}$




$\begin{array}{lrrr}\mathrm{H} & -1.58897300 & 1.40417700 & -4.70980100 \\ \mathrm{H} & -1.66149300 & 3.75021200 & 3.12777400 \\ \mathrm{H} & -1.27604900 & -1.76244400 & 4.88464500 \\ \mathrm{H} & -1.22267800 & -4.20723000 & -3.02867300 \\ \mathrm{O} & -4.82855300 & -1.65914700 & 1.84576400 \\ \mathrm{H} & -4.33295900 & -2.51395300 & 2.07218400 \\ \mathrm{H} & -4.05634100 & -0.47629400 & 2.36462400 \\ \mathrm{O} & -3.23052500 & -3.69154300 & 2.39076300 \\ \mathrm{H} & -3.18004900 & -3.85093700 & 1.43608700 \\ \mathrm{H} & -2.37576700 & -3.27251900 & 2.60865700\end{array}$

$\begin{array}{lrrr}\mathrm{Al}_{2} @ N U-1000 \mathrm{TS} 2 & & \\ \mathrm{C} & -0.50317900 & -1.45586100 & 4.14275200 \\ \mathrm{C} & 4.07166900 & -0.74512500 & 3.88211300 \\ \mathrm{C} & 3.40780400 & 3.49438200 & 2.28866500 \\ \mathrm{C} & 3.09618400 & 1.38653000 & -4.06503700 \\ \mathrm{C} & -1.31276000 & 0.80251400 & -3.74878100 \\ \mathrm{C} & -0.57059900 & -3.68360200 & -2.25930100 \\ \mathrm{C} & -1.26816800 & 2.91857100 & 2.56726100 \\ \mathrm{C} & 3.75632300 & -3.21709400 & -2.77434000 \\ \mathrm{H} & 1.80707500 & -2.07270200 & 2.79851700 \\ \mathrm{H} & 5.40626700 & 2.37920600 & 0.16929800 \\ \mathrm{H} & 5.38296100 & 0.83964500 & -0.23591600 \\ \mathrm{H} & 0.46161400 & 3.79435300 & -1.41359900 \\ \mathrm{H} & 2.47048700 & 4.02294700 & -2.26970800 \\ \mathrm{H} & -1.09286900 & 3.88179700 & -1.91308700 \\ \mathrm{H} & 4.25115000 & -0.28196300 & -1.95598200 \\ \mathrm{H} & 0.84087400 & 3.23769100 & 0.87260100 \\ \mathrm{H} & 6.05886400 & -0.96569000 & 0.93415200 \\ \mathrm{H} & 3.73880800 & -3.88589300 & 0.11538800 \\ \mathrm{H} & 0.51045300 & -4.82431600 & 0.97673400 \\ \mathrm{H} & 2.54118600 & -3.85285500 & 1.14724600 \\ \mathrm{O} & 2.77053200 & 0.58858000 & 1.02108100 \\ \mathrm{O} & 2.68777900 & 2.78444800 & 3.03736700 \\ \mathrm{O} & -0.29536600 & 2.30573200 & 3.10526600 \\ \mathrm{O} & 3.05801000 & -0.02138500 & 4.02739200 \\ \mathrm{O} & 4.51753900 & -1.26733400 & 2.82733500 \\ \mathrm{O} & 3.48104800 & -3.52290700 & 0.99097400 \\ \mathrm{O} & 4.12029100 & -3.32026400 & -1.58856900 \\ \mathrm{O} & -0.71310400 & -3.67940900 & -1.00854000 \\ \mathrm{O} & 2.85573100 & -2.46635900 & -3.25598000 \\ \mathrm{O} & 0.15323200 & -2.88049600 & -2.91927000 \\ \mathrm{O} & 2.33305800 & 0.39541500 & -4.06235500 \\ \mathrm{O} & -0.46725400 & -0.10590600 & -3.92527900\end{array}$




\begin{tabular}{|c|c|c|c|}
\hline $\mathrm{O}$ & -1.51526300 & 1.43398900 & -2.66359100 \\
\hline $\mathrm{O}$ & 3.46959000 & 2.08749900 & -3.08750100 \\
\hline $\mathrm{O}$ & 1.17485100 & 0.95017300 & -1.52286500 \\
\hline $\mathrm{O}$ & 3.44322000 & -0.19176100 & -1.43151000 \\
\hline $\mathrm{O}$ & 5.43905500 & -0.76862900 & 0.21928200 \\
\hline $\mathrm{O}$ & 5.08926400 & 1.76529000 & -0.50857300 \\
\hline $\mathrm{O}$ & -2.01224900 & -1.89768400 & 0.72769500 \\
\hline $\mathrm{O}$ & 1.71667600 & -1.78433600 & -0.56164100 \\
\hline $\mathrm{O}$ & 1.76011300 & -1.47607800 & 2.03849600 \\
\hline $\mathrm{O}$ & -0.59491000 & -2.20605000 & 3.13188800 \\
\hline $\mathrm{O}$ & 0.89221100 & -3.98485700 & 1.25671300 \\
\hline $\mathrm{O}$ & 0.06797600 & -0.32961600 & 4.19673200 \\
\hline $\mathrm{O}$ & 2.12743900 & 3.76737800 & -1.40298600 \\
\hline $\mathrm{O}$ & -0.48577100 & 3.50407500 & -1.24747200 \\
\hline $\mathrm{O}$ & 1.02339000 & 2.31103600 & 0.66095900 \\
\hline $\mathrm{O}$ & 3.57920400 & 3.40761500 & 1.04911500 \\
\hline $\mathrm{O}$ & -1.64160000 & 2.80058200 & 1.37032600 \\
\hline $\mathrm{O}$ & -2.46744900 & 0.51180200 & -0.07659100 \\
\hline $\mathrm{O}$ & -0.58125200 & -0.83499600 & -1.15112700 \\
\hline $\mathrm{O}$ & -0.25413700 & 0.10145000 & 1.36543000 \\
\hline $\mathrm{Zr}$ & 1.28432500 & -0.96362000 & -2.45194600 \\
\hline $\mathrm{Zr}$ & 3.47350900 & -1.30739400 & 0.54972600 \\
\hline $\mathrm{Zr}$ & 2.81974000 & 1.94007900 & -0.74236500 \\
\hline $\mathrm{Zr}$ & -0.63459600 & 1.35277800 & -0.58955400 \\
\hline $\mathrm{Zr}$ & 1.38989200 & 0.84734200 & 2.56215900 \\
\hline $\mathrm{Zr}$ & 0.06980800 & -2.19803400 & 0.63814100 \\
\hline $\mathrm{O}$ & -3.29865600 & -0.14892900 & 2.73551200 \\
\hline $\mathrm{H}$ & -3.74410200 & -1.04639200 & 2.77819400 \\
\hline $\mathrm{O}$ & -2.76239800 & -1.69754100 & -2.62715900 \\
\hline $\mathrm{H}$ & -1.99044500 & -1.65338100 & -3.20016300 \\
\hline $\mathrm{Al}$ & -2.49179400 & -1.18297400 & -0.98894200 \\
\hline $\mathrm{Al}$ & -1.99573900 & -0.27659800 & 1.43716400 \\
\hline $\mathrm{H}$ & 3.49217500 & 1.70330700 & -5.06043800 \\
\hline $\mathrm{H}$ & 4.27633400 & -3.85683900 & -3.53344900 \\
\hline $\mathrm{H}$ & 3.96582800 & 4.31583100 & 2.80064900 \\
\hline $\mathrm{H}$ & 4.65877600 & -0.95531400 & 4.80871300 \\
\hline $\mathrm{O}$ & -4.30790100 & -1.39132500 & -0.58073000 \\
\hline $\mathrm{P}$ & -5.34805800 & -1.78136100 & 0.46800200 \\
\hline $\mathrm{O}$ & -6.91373600 & -1.70098500 & 0.09720600 \\
\hline $\mathrm{O}$ & -5.32921200 & -3.43821000 & 0.29685900 \\
\hline $\mathrm{O}$ & -5.41734200 & 0.02949500 & 1.12784800 \\
\hline $\mathrm{C}$ & -7.81562100 & -0.61420000 & 0.20224000 \\
\hline $\mathrm{H}$ & -8.78986800 & -1.00139500 & -0.11701800 \\
\hline $\mathrm{H}$ & -7.89176900 & -0.25004900 & 1.23401800 \\
\hline
\end{tabular}




$\begin{array}{lrrr}\mathrm{H} & -7.53976200 & 0.22232700 & -0.45422900 \\ \mathrm{C} & -5.42615300 & -4.01751600 & -0.98665600 \\ \mathrm{H} & -5.26249700 & -5.09590700 & -0.87408900 \\ \mathrm{H} & -6.42578500 & -3.85909200 & -1.42157600 \\ \mathrm{H} & -4.66872100 & -3.60910100 & -1.67381400 \\ \mathrm{C} & -5.21762600 & 1.15910700 & 0.45377600 \\ \mathrm{C} & -4.98370900 & 2.31070200 & 1.24193200 \\ \mathrm{C} & -5.15020900 & 1.28214200 & -0.95369900 \\ \mathrm{C} & -4.46851300 & 3.46116500 & 0.68312000 \\ \mathrm{H} & -5.14579100 & 2.24622200 & 2.32260000 \\ \mathrm{C} & -4.59952000 & 2.42149000 & -1.51219800 \\ \mathrm{H} & -5.39831000 & 0.43635600 & -1.59370800 \\ \mathrm{C} & -4.20414000 & 3.48284100 & -0.68885700 \\ \mathrm{H} & -4.19395900 & 4.32107100 & 1.29296000 \\ \mathrm{H} & -4.42119400 & 2.48552600 & -2.58611900 \\ \mathrm{~N} & -3.41381900 & 4.56538800 & -1.21866200 \\ \mathrm{O} & -2.91536300 & 4.41643700 & -2.34374300 \\ \mathrm{O} & -3.22063900 & 5.54942800 & -0.52207800 \\ \mathrm{H} & -1.91558300 & 1.08484100 & -4.58646300 \\ \mathrm{H} & -1.79435900 & 3.60626700 & 3.19585200 \\ \mathrm{H} & -0.94430900 & -1.82328600 & 5.04568400 \\ \mathrm{H} & -1.08115400 & -4.44634200 & -2.80933500 \\ \mathrm{O} & -4.96055100 & -2.02776600 & 2.07007000 \\ \mathrm{H} & -4.85166800 & -2.99301600 & 2.13255700 \\ \mathrm{H} & -4.08146300 & 0.33019900 & 2.34497200\end{array}$

$\begin{array}{lrrr}\mathrm{Al}_{2} @ N U-1000 \text { DMPA Binding } & \\ \mathrm{C} & -1.70951700 & 3.57137900 & 1.68611500 \\ \mathrm{C} & 2.78459600 & 3.36407000 & 2.80966800 \\ \mathrm{C} & 3.96555000 & 3.13940600 & -1.60711000 \\ \mathrm{C} & 3.40680600 & -3.53754700 & -1.73284200 \\ \mathrm{C} & -0.89058600 & -3.24544900 & -2.88454900 \\ \mathrm{C} & -2.08684900 & -3.19673000 & 1.74800600 \\ \mathrm{C} & -0.57023700 & 3.40805500 & -2.88318600 \\ \mathrm{C} & 2.10678100 & -3.71044700 & 2.91209400 \\ \mathrm{H} & 0.27423400 & 2.04154500 & 2.74207400 \\ \mathrm{H} & 5.52929600 & 0.72561000 & -0.44742600 \\ \mathrm{H} & 4.95705600 & -0.13183500 & 0.76641600 \\ \mathrm{H} & 1.71253400 & -0.21231800 & -4.03241300 \\ \mathrm{H} & 3.70975200 & -1.00561700 & -3.74236800 \\ \mathrm{H} & 0.43281600 & -0.93609500 & -4.65978600 \\ \mathrm{H} & 3.63484700 & -2.06239700 & 0.79481300 \\ \mathrm{H} & 1.64179300 & 1.82843000 & -2.80141600 \\ \mathrm{H} & 4.77324700 & 0.40954200 & 2.94471100\end{array}$




\begin{tabular}{|c|c|c|c|}
\hline $\mathrm{H}$ & 1.55945800 & -1.15032000 & \\
\hline $\mathrm{H}$ & -1.78201500 & -0.58794400 & 4.05596300 \\
\hline $\mathrm{H}$ & 0.38773000 & -0.10861800 & 4.09856900 \\
\hline & 2.35139900 & 1.08977500 & 0.30506100 \\
\hline C & 2.96410000 & 3.66740900 & -1.05836300 \\
\hline $\mathrm{C}$ & 0.03639100 & 3.70077100 & -1.80726800 \\
\hline C & 2.12456500 & 3.75759500 & 1.81859800 \\
\hline C & 3.08096900 & 2.18534700 & 3.13657400 \\
\hline C & 1.39184800 & -0.19650200 & 4.15244300 \\
\hline C & 2.29791900 & -2.62451300 & 3.48951400 \\
\hline $\mathrm{C}$ & -2.32375000 & -1.99748400 & 2.04949400 \\
\hline $\mathrm{C}$ & 1.61508300 & -3.91106900 & 1.76085500 \\
\hline $\mathrm{C}$ & -1.05315300 & -3.61257600 & 1.14512300 \\
\hline $\mathrm{C}$ & 2.32065200 & -3.80319300 & -1.17229600 \\
\hline C & -0.45412900 & -3.71750700 & -1.80855400 \\
\hline $\mathrm{C}$ & -0.92375700 & -2.01430300 & -3.20077800 \\
\hline $\mathrm{C}$ & 3.93797700 & -2.40969000 & -1.91209500 \\
\hline $\mathrm{O}$ & 1.25181800 & -1.16984900 & -1.38125600 \\
\hline $\mathrm{O}$ & 2.88164800 & -1.50863500 & 0.54589200 \\
\hline $\mathrm{C}$ & 4.34329800 & -0.18691400 & 2.31750500 \\
\hline $\mathrm{O}$ & 5.07066700 & -0.10028100 & -0.23573700 \\
\hline $\mathrm{O}$ & -2.96044100 & 0.26557700 & 0.48040600 \\
\hline $\mathrm{O}$ & 0.60634500 & -1.08015600 & 51200 \\
\hline $\mathrm{O}$ & 0.53761000 & 1.48949500 & 1.99243300 \\
\hline $\mathrm{O}$ & -1.99867900 & 2.38643600 & 2.01069400 \\
\hline $\mathrm{O}$ & -1.18657500 & 0.01184600 & 3.59266100 \\
\hline $\mathrm{O}$ & -0.75166000 & 3.93880800 & 0.94783200 \\
\hline $\mathrm{O}$ & 3.20747300 & -0.24700800 & -3.41628600 \\
\hline $\mathrm{O}$ & 0.70914100 & -0.15138300 & -4.16681500 \\
\hline $\mathrm{O}$ & 1.45510600 & 1.32822000 & -1.99487500 \\
\hline $\mathrm{O}$ & 4.19587000 & 1.92525400 & -1.82195600 \\
\hline $\mathrm{O}$ & -0.85535200 & 2.24562000 & -3.27494800 \\
\hline $\mathrm{O}$ & -2.40874900 & 0.20093300 & -2.03041200 \\
\hline $\mathrm{O}$ & -1.08517100 & -1.28179900 & -0.41208300 \\
\hline $\mathrm{O}$ & -0.64496000 & 1.37340100 & -0.38580500 \\
\hline $\mathrm{Zr}$ & 0.68893000 & -2.62615200 & 0.06680200 \\
\hline $\mathrm{Zr}$ & 2.30211300 & 0.05114700 & 2.10042500 \\
\hline $\mathrm{Zr}$ & 3.07277000 & -0.18653600 & -1.34920200 \\
\hline $\mathrm{Zr}$ & -0.35204800 & -0.07015800 & -2.14298600 \\
\hline $\mathrm{Zr}$ & 1.03749100 & 2.68928900 & -0.02203500 \\
\hline $\mathrm{Zr}$ & -1.16973400 & 0.02272400 & 1.52301200 \\
\hline $\mathrm{O}$ & -3.51587400 & 2.81273000 & -0.85231200 \\
\hline $\mathrm{H}$ & -3.53114400 & 3.44430100 & -1.57469400 \\
\hline $\mathrm{O}$ & -3.34125400 & -2.81701600 & -0.94727700 \\
\hline
\end{tabular}




$\begin{array}{lrrr}\mathrm{H} & -2.57781200 & -3.39699100 & -0.86507000 \\ \mathrm{Al} & -2.98749900 & -1.12679600 & -0.82161600 \\ \mathrm{Al} & -2.43480100 & 1.46936800 & -0.74768000 \\ \mathrm{H} & 3.97926600 & -4.40651700 & -2.13919300 \\ \mathrm{H} & 2.39974700 & -4.64524500 & 3.45636500 \\ \mathrm{H} & 4.75309000 & 3.85258600 & -1.95276400 \\ \mathrm{H} & 3.16105800 & 4.16247100 & 3.49396400 \\ \mathrm{O} & -4.83511700 & -0.62511700 & -1.06554800 \\ \mathrm{P} & -5.82888100 & 0.03930600 & -0.14804500 \\ \mathrm{O} & -7.27588500 & -0.31664400 & -0.70061200 \\ \mathrm{O} & -5.89659600 & -0.49087400 & 1.35206700 \\ \mathrm{O} & -5.76704600 & 1.61804100 & -0.05043800 \\ \mathrm{C} & -8.44444300 & 0.15308600 & -0.04306900 \\ \mathrm{H} & -9.30004200 & -0.18743700 & -0.63438700 \\ \mathrm{H} & -8.51811300 & -0.26258900 & 0.97152000 \\ \mathrm{H} & -8.45625900 & 1.25118500 & 0.00865200 \\ \mathrm{C} & -5.16839000 & 0.04255100 & 2.45948400 \\ \mathrm{H} & -5.84698600 & 0.03774100 & 3.32041800 \\ \mathrm{H} & -4.30234600 & -0.59778800 & 2.66638800 \\ \mathrm{H} & -4.82635100 & 1.06641000 & 2.26303800 \\ \mathrm{H} & -4.95901900 & 2.10365400 & -0.38776900 \\ \mathrm{H} & -0.83898000 & 4.23300800 & -3.50938000 \\ \mathrm{H} & -1.26098800 & -3.93691200 & -3.61229200 \\ \mathrm{H} & -2.80467600 & -3.93035800 & 2.05031600 \\ \mathrm{H} & -2.33510600 & 4.33874000 & 2.09195900\end{array}$

$\begin{array}{lrrr}\mathrm{Al}_{2} @ \mathrm{NU}-1000+1 \mathrm{~W} \text { TS } 1 & & \\ \mathrm{C} & -1.31222000 & 1.24048100 & 3.64009000 \\ \mathrm{C} & 3.25362800 & 1.97053200 & 3.99009400 \\ \mathrm{C} & 3.25211200 & 4.38150100 & 0.09902800 \\ \mathrm{C} & 4.13844900 & -1.09843300 & -3.65524600 \\ \mathrm{C} & -0.26818700 & -1.68245900 & -4.00174300 \\ \mathrm{C} & -0.18640900 & -4.34753400 & -0.02873500 \\ \mathrm{C} & -1.40624700 & 3.76340500 & -0.33847800 \\ \mathrm{C} & 4.17385600 & -3.98129900 & 0.21554000 \\ \mathrm{H} & 1.17222400 & 0.11516500 & 3.44202100 \\ \mathrm{H} & 5.56634200 & 2.38173100 & -0.46117900 \\ \mathrm{H} & 5.52844500 & 0.90522000 & 0.13234300 \\ \mathrm{H} & 1.23002000 & 2.18165900 & -3.53760300 \\ \mathrm{H} & 3.35484900 & 2.05354400 & -3.94602400 \\ \mathrm{H} & 0.01077900 & 1.52086700 & -4.33483600 \\ \mathrm{H} & 4.72006900 & -1.08566100 & -0.77960200 \\ \mathrm{H} & 1.04293700 & 3.17577100 & -1.38467000 \\ \mathrm{H} & 5.80753000 & 0.19234100 & 2.25818800\end{array}$




\begin{tabular}{|c|c|c|c|}
\hline $\mathrm{H}$ & 3.44601100 & -2.80579400 & 2.84138200 \\
\hline $\mathrm{H}$ & 0.13482300 & -3.30585600 & 3.22380600 \\
\hline $\mathrm{H}$ & 2.06576200 & -2.22573700 & 3.35643000 \\
\hline $\mathrm{O}$ & 2.68833200 & 1.25928200 & 0.69809500 \\
\hline $\mathrm{O}$ & 2.34038200 & 4.20627900 & 0.94811800 \\
\hline $\mathrm{O}$ & -0.61645800 & 3.65746700 & 0.64963600 \\
\hline $\mathrm{O}$ & 2.28757400 & 2.56772400 & 3.45855900 \\
\hline $\mathrm{O}$ & 3.87322100 & 0.95677700 & 3.57483000 \\
\hline $\mathrm{O}$ & 3.04377200 & -2.00852300 & 3.25053300 \\
\hline $\mathrm{O}$ & 4.27035100 & -3.33597400 & 1.27545700 \\
\hline $\mathrm{O}$ & -0.58931900 & -3.61222700 & 0.91030800 \\
\hline $\mathrm{O}$ & 3.45254900 & -3.72645300 & -0.79509700 \\
\hline $\mathrm{O}$ & 0.71717600 & -4.04476900 & -0.86324600 \\
\hline $\mathrm{O}$ & 3.32197200 & -1.94449700 & -3.22836300 \\
\hline $\mathrm{O}$ & 0.52707200 & -2.45784100 & -3.42078000 \\
\hline $\mathrm{O}$ & -0.64917800 & -0.54622900 & -3.57596500 \\
\hline $\mathrm{O}$ & 4.34674400 & 0.06919200 & -3.23148100 \\
\hline $\mathrm{O}$ & 1.70290400 & -0.07172200 & -1.83171300 \\
\hline $\mathrm{O}$ & 3.82397600 & -0.76891200 & -0.59904500 \\
\hline $\mathrm{O}$ & 5.36841400 & -0.11377000 & 1.45342500 \\
\hline $\mathrm{O}$ & 5.36376200 & 1.46337000 & -0.69095700 \\
\hline $\mathrm{O}$ & -2.07154700 & -1.20797900 & 0.92919800 \\
\hline $\mathrm{O}$ & 1.84472300 & -1.64998200 & 0.63497300 \\
\hline $\mathrm{O}$ & 1.33465500 & 0.14682700 & 2.48896800 \\
\hline $\mathrm{O}$ & -1.24248300 & 0.03290800 & 3.27961500 \\
\hline $\mathrm{O}$ & 0.40326100 & -2.38176100 & 3.15962800 \\
\hline $\mathrm{O}$ & -0.68497200 & 2.21537100 & 3.13638900 \\
\hline $\mathrm{O}$ & 2.80294400 & 2.32479800 & -3.20054800 \\
\hline $\mathrm{O}$ & 0.23851400 & 1.96870500 & -3.50908800 \\
\hline $\mathrm{O}$ & 1.18881900 & 2.31147600 & -0.97468900 \\
\hline $\mathrm{O}$ & 3.67498200 & 3.58866300 & -0.77620400 \\
\hline $\mathrm{O}$ & -1.52572500 & 2.93334600 & -1.27804300 \\
\hline $\mathrm{O}$ & -2.19818000 & 0.17679100 & -1.23709500 \\
\hline $\mathrm{O}$ & -0.21887500 & -1.40797000 & -0.86187100 \\
\hline $\mathrm{O}$ & -0.36943900 & 0.85967600 & 0.59160700 \\
\hline $\mathrm{Zr}$ & 1.86876800 & -2.15259900 & -1.40136200 \\
\hline $\mathrm{Zr}$ & 3.34415700 & -0.48425200 & 1.60765500 \\
\hline $\mathrm{Zr}$ & 3.20961100 & 1.29615100 & -1.44911600 \\
\hline $\mathrm{Zr}$ & -0.24161500 & 0.67809500 & -1.68887900 \\
\hline $\mathrm{Zr}$ & 1.02097600 & 2.28915600 & 1.44443300 \\
\hline $\mathrm{Zr}$ & -0.05162000 & -1.34191200 & 1.42331700 \\
\hline $\mathrm{O}$ & -3.45019400 & 1.29439000 & 1.42665900 \\
\hline $\mathrm{H}$ & -3.83280000 & 2.15338400 & 1.22014800 \\
\hline $\mathrm{O}$ & -2.14165700 & -3.11708100 & -1.90191700 \\
\hline
\end{tabular}




$\begin{array}{llrc}\mathrm{H} & -1.27006900 & -3.36798800 & -2.22418800 \\ \mathrm{Al} & -2.17763500 & -1.69410000 & -0.90492700 \\ \mathrm{Al} & -2.17967000 & 0.54476800 & 0.52573700 \\ \mathrm{H} & 4.75794500 & -1.40728700 & -4.53206100 \\ \mathrm{H} & 4.79463000 & -4.90895000 & 0.11541600 \\ \mathrm{H} & 3.74753500 & 5.38246900 & 0.12890700 \\ \mathrm{H} & 3.61462800 & 2.39166300 & 4.95953200 \\ \mathrm{O} & -4.05982600 & -1.68716800 & -0.76389200 \\ \mathrm{P} & -5.12367800 & -1.95262100 & 0.31373400 \\ \mathrm{O} & -6.30377800 & -3.01633400 & -0.01328800 \\ \mathrm{O} & -4.37674900 & -3.10525500 & 1.26990000 \\ \mathrm{O} & -6.23505600 & -0.68879200 & -0.14643100 \\ \mathrm{C} & -7.32256000 & -2.84090200 & -0.98330900 \\ \mathrm{H} & -7.71458600 & -3.84279500 & -1.19688700 \\ \mathrm{H} & -8.13133900 & -2.20621800 & -0.60176100 \\ \mathrm{H} & -6.93192900 & -2.40219700 & -1.91097300 \\ \mathrm{C} & -4.12105500 & -4.38934700 & 0.72882300 \\ \mathrm{H} & -3.36635200 & -4.86287300 & 1.37097800 \\ \mathrm{H} & -5.02675100 & -5.01171400 & 0.72538900 \\ \mathrm{H} & -3.71540900 & -4.32586500 & -0.29460400 \\ \mathrm{C} & -5.92844500 & 0.59348500 & -0.39528100 \\ \mathrm{C} & -6.47458100 & 1.58384100 & 0.44357400 \\ \mathrm{H} & -5.10487300 & 0.96741300 & -1.47083900 \\ \mathrm{H} & -2.94750100 & -2.51320200 & 2.84256000 \\ \mathrm{C} & -6.15655400 & 2.91956100 & 0.25114200 \\ \mathrm{H} & -7.13268000 & 1.27153200 & 1.25695100 \\ \mathrm{C} & -4.77507300 & 2.30121300 & -1.66096100 \\ \mathrm{H} & -4.69878900 & 0.19693600 & -2.12250700 \\ \mathrm{C} & -5.28638300 & 3.26297200 & -0.78784700 \\ \mathrm{H} & -6.55213500 & 3.70459800 & 0.89619000 \\ \mathrm{H} & -4.10439600 & 2.60960600 & -2.46274800 \\ \mathrm{H} & -4.89389100 & 4.66297900 & -0.95325000 \\ \mathrm{O} & -4.16704400 & 4.94234900 & -1.89588600 \\ \mathrm{H} & -5.29917500 & 5.46646100 & -0.12548200 \\ \mathrm{H} & -0.65808500 & -1.99406000 & -4.94819900 \\ \mathrm{H} & -2.00197200 & 4.65168900 & -0.36947800 \\ \mathrm{H} & -1.95985200 & 1.45076300 & 4.46547800 \\ \mathrm{H} & -4.62321200 & -5.32069500 & -0.11286000 \\ \mathrm{H} & -3.618248000 & -1.35589700 & 2.54494100 \\ \mathrm{H} & & -1.16794100 & 3.49333800\end{array}$




$\begin{array}{lrrr}\mathrm{Al}_{2} @ \mathrm{NU}-1000+1 \mathrm{~W} \mathrm{TS} 2 & & \\ \mathrm{C} & -0.81328700 & -1.22669600 & 4.05357900 \\ \mathrm{C} & 3.80831300 & -0.84871100 & 4.03771200 \\ \mathrm{C} & 3.54061700 & 3.38800500 & 2.32546400 \\ \mathrm{C} & 3.42711700 & 1.15666400 & -3.99259800 \\ \mathrm{C} & -1.02317300 & 0.89460100 & -3.91435900 \\ \mathrm{C} & -0.68958900 & -3.59606600 & -2.29657400 \\ \mathrm{C} & -1.17294100 & 3.15216900 & 2.35086500 \\ \mathrm{C} & 3.68145400 & -3.45010300 & -2.57554100 \\ \mathrm{H} & 1.51816000 & -2.03060700 & 2.84494000 \\ \mathrm{H} & 5.57912500 & 2.10034400 & 0.33824300 \\ \mathrm{H} & 5.46763300 & 0.55652600 & -0.03181100 \\ \mathrm{H} & 0.81513400 & 3.87744200 & -1.52770000 \\ \mathrm{H} & 2.90053300 & 3.86565800 & -2.30020300 \\ \mathrm{H} & -0.80017800 & 4.17478200 & -1.82211000 \\ \mathrm{H} & 4.36008400 & -0.52201800 & -1.78740500 \\ \mathrm{H} & 1.05118600 & 3.28275700 & 0.76118700 \\ \mathrm{H} & 5.95202400 & -1.27518900 & 1.20933900 \\ \mathrm{H} & 3.43107300 & -4.04087200 & 0.31845200 \\ \mathrm{H} & 0.13188200 & -4.69636900 & 0.91249200 \\ \mathrm{H} & 2.18880900 & -3.88343600 & 1.28397600 \\ \mathrm{O} & 2.77733500 & 0.51053800 & 1.08169200 \\ \mathrm{O} & 2.73121200 & 2.74899500 & 3.04624500 \\ \mathrm{O} & -0.27791600 & 2.48486600 & 2.95502000 \\ \mathrm{O} & 2.84307700 & -0.05162600 & 4.11110600 \\ \mathrm{O} & 4.27259000 & -1.42623700 & 3.02037800 \\ \mathrm{O} & 3.15738200 & -3.63568500 & 1.17054600 \\ \mathrm{O} & 3.97135500 & -3.55052800 & -1.36929000 \\ \mathrm{O} & -0.89989500 & -3.55195100 & -1.05618900 \\ \mathrm{O} & 0.50536000 & -3.89420200 & 1.29563900 \\ \mathrm{O} & 2.86519700 & -2.64906900 & -3.12209000 \\ \mathrm{O} & 0.12545600 & -2.86229900 & -2.93041600 \\ \mathrm{O} & 2.59546900 & 0.22252900 & -4.01335200 \\ \mathrm{O} & -0.23700900 & -0.07546100 & -4.02474700 \\ \mathrm{O} & -1.23887200 & 1.56454700 & -2.85508900 \\ \mathrm{O} & 3.79588100 & 1.85248700 & -3.00963900 \\ \mathrm{O} & 1.34752700 & 0.92466500 & -1.56042100 \\ \mathrm{O} & 3.53047300 & -0.36866800 & -1.31388700 \\ \mathrm{O} & 5.38376200 & -1.04881700 & 0.46106700 \\ \mathrm{O} & -25387900 & 1.49144200 & -0.34028400 \\ \mathrm{O} & 1.65368500 & -1.81035800 & -0.52425100 \\ \mathrm{O} & & -1.61444000 & 0.58159000 \\ \mathrm{O} & -90325900 & -1.99237800 & 3.05423900 \\ \mathrm{O} & & & \\ \mathrm{O} & & & \end{array}$




\begin{tabular}{|c|c|c|c|}
\hline $\mathrm{O}$ & -0.16618000 & -0.14284000 & 4.11736700 \\
\hline $\mathrm{O}$ & 2.50471900 & 3.66998800 & -1.44023500 \\
\hline $\mathrm{O}$ & -0.13392600 & 3.61442800 & -1.36470200 \\
\hline $\mathrm{C}$ & 1.18436600 & 2.33823500 & 0.59606400 \\
\hline $\mathrm{C}$ & 3.77312500 & 3.25976500 & 1.09954200 \\
\hline $\mathrm{O}$ & -1.48761300 & 3.03246100 & 1.13732600 \\
\hline $\mathrm{O}$ & -2.38419000 & 0.78459000 & -0.34138300 \\
\hline $\mathrm{C}$ & -0.54513200 & -0.73207800 & -1.25682000 \\
\hline $\mathrm{O}$ & -0.28892900 & 0.25689300 & 1.25012800 \\
\hline $\mathrm{Zr}$ & 1.37012900 & -1.01337600 & -2.45224100 \\
\hline $\mathrm{Zr}$ & 3.37101000 & -1.43594100 & 0.68726700 \\
\hline $\mathrm{Zr}$ & 3.01688200 & 1.81594200 & -0.70599400 \\
\hline $\mathrm{Zr}$ & -0.47181600 & 1.49415100 & -0.74900000 \\
\hline $\mathrm{Zr}$ & 1.33426400 & 0.91147800 & 2.53009200 \\
\hline $\mathrm{Zr}$ & -0.08007400 & -2.04735500 & 0.57663500 \\
\hline $\mathrm{O}$ & -3.36455900 & 0.40344800 & 2.45746900 \\
\hline $\mathrm{H}$ & -4.08636300 & -0.32096700 & 2.33778600 \\
\hline $\mathrm{O}$ & -2.71228700 & -1.48523300 & -2.81985600 \\
\hline $\mathrm{H}$ & -1.91851900 & -1.49726400 & -3.36431100 \\
\hline $\mathrm{Al}$ & -2.48250900 & -0.92205100 & -1.19195000 \\
\hline $\mathrm{Al}$ & -2.06189100 & 0.03309700 & 1.23080500 \\
\hline $\mathrm{H}$ & 3.89900200 & 1.42072500 & -4.97013400 \\
\hline $\mathrm{H}$ & 4.19484800 & -4.14304400 & -3.29121400 \\
\hline $\mathrm{H}$ & 4.12750100 & 4.17977300 & 2.85174300 \\
\hline $\mathrm{H}$ & 4.32694500 & -1.07786500 & 4.99998500 \\
\hline $\mathrm{O}$ & -4.32334800 & -1.06364400 & -0.81940500 \\
\hline $\mathrm{P}$ & -5.19601600 & -1.69677100 & 0.26440100 \\
\hline $\mathrm{O}$ & -6.74197300 & -1.91976500 & -0.06466400 \\
\hline $\mathrm{O}$ & -4.79741800 & -3.29534300 & 0.17382500 \\
\hline $\mathrm{O}$ & -5.74303600 & 0.28132100 & 0.82626100 \\
\hline $\mathrm{C}$ & -7.71247400 & -0.95277600 & -0.43309200 \\
\hline $\mathrm{H}$ & -8.54017700 & -1.51637300 & -0.87889600 \\
\hline $\mathrm{H}$ & -8.06870100 & -0.39813400 & 0.44189600 \\
\hline $\mathrm{H}$ & -7.32217000 & -0.24348600 & -1.17392200 \\
\hline $\mathrm{C}$ & -4.82419700 & -3.93318700 & -1.09156000 \\
\hline $\mathrm{H}$ & -4.44870300 & -4.95365700 & -0.94966000 \\
\hline $\mathrm{H}$ & -5.85043600 & -3.98931200 & -1.48521700 \\
\hline $\mathrm{H}$ & -4.18601500 & -3.41045600 & -1.82255600 \\
\hline $\mathrm{C}$ & -5.36712700 & 1.40331200 & 0.31142200 \\
\hline $\mathrm{C}$ & -5.10527400 & 2.50090600 & 1.19953100 \\
\hline $\mathrm{C}$ & -5.13655200 & 1.63768400 & -1.07820100 \\
\hline $\mathrm{C}$ & -4.43389300 & 3.63048700 & 0.77406600 \\
\hline $\mathrm{H}$ & -5.44984200 & 2.41063500 & 2.23619500 \\
\hline $\mathrm{C}$ & -4.44557300 & 2.75274300 & -1.49636200 \\
\hline
\end{tabular}




$\begin{array}{lrrr}\mathrm{H} & -5.40131800 & 0.86694500 & -1.80130600 \\ \mathrm{C} & -4.03101500 & 3.71926800 & -0.56383600 \\ \mathrm{H} & -4.15788300 & 4.43003400 & 1.46293700 \\ \mathrm{H} & -4.16656200 & 2.87753400 & -2.54360500 \\ \mathrm{~N} & -3.08584100 & 4.72362900 & -0.93428600 \\ \mathrm{O} & -2.53544500 & 4.61402700 & -2.04930700 \\ \mathrm{O} & -2.80765300 & 5.61293300 & -0.14257800 \\ \mathrm{H} & -1.55713500 & 1.19892500 & -4.79019300 \\ \mathrm{H} & -1.68186000 & 3.89025400 & 2.93495500 \\ \mathrm{H} & -1.32870200 & -1.54026500 & 4.93726800 \\ \mathrm{H} & -1.22287400 & -4.33356400 & -2.85931800 \\ \mathrm{O} & -4.80683200 & -1.63379400 & 1.84479300 \\ \mathrm{H} & -4.27122000 & -2.45431000 & 2.12948100 \\ \mathrm{H} & -3.83209800 & 1.25144500 & 2.25546100 \\ \mathrm{O} & -3.21597300 & -3.59703700 & 2.53134100 \\ \mathrm{H} & -2.36406900 & -3.16461000 & 2.74161200 \\ \mathrm{H} & -3.12830000 & -3.86388300 & 1.60442600\end{array}$

\section{References}

(1) Chen, H.; Liao, P.; Mendonca, M. L.; Snurr, R. Q. Insights into Catalytic Hydrolysis of Organophosphate Warfare Agents by Metal-Organic Framework NU-1000. J. Phys. Chem. C 2018, $122,12362-12368$. 